\title{
Evolving therapies for liver fibrosis
}

\author{
Detlef Schuppan ${ }^{1,2}$ and Yong Ook Kim¹
}

\begin{abstract}
${ }^{1}$ Institute of Molecular and Translational Medicine and Department of Medicine I, University Medical Center, Johannes Gutenberg University Mainz, Mainz, Germany. 2Beth Israel Deaconess Medical Center, Harvard Medical School, Boston, Massachusetts, USA.
\end{abstract}

\begin{abstract}
Fibrosis is an intrinsic response to chronic injury, maintaining organ integrity when extensive necrosis or apoptosis occurs. With protracted damage, fibrosis can progress toward excessive scarring and organ failure, as in liver cirrhosis. To date, antifibrotic treatment of fibrosis represents an unconquered area for drug development, with enormous potential but also high risks. Preclinical research has yielded numerous targets for antifibrotic agents, some of which have entered early-phase clinical studies, but progress has been hampered due to the relative lack of sensitive and specific biomarkers to measure fibrosis progression or reversal. Here we focus on antifibrotic approaches for liver that address specific cell types and functional units that orchestrate fibrotic wound healing responses and have a sound preclinical database or antifibrotic activity in early clinical trials. We also touch upon relevant clinical study endpoints, optimal study design, and developments in fibrosis imaging and biomarkers.
\end{abstract}

\section{The clinical problem}

Fibrosis is the excess accumulation of ECM, which results from chronic, nonresolving inflammation. This inflammation triggers a wound-healing process that mitigates inflammatory tissue destruction but also leads to scar tissue formation. In the liver, fibrosis is mainly due to chronic viral hepatitis $\mathrm{B}$ or $\mathrm{C}$, autoimmune and biliary diseases, alcoholic steatohepatitis (ASH) and, increasingly, nonalcoholic steatohepatitis (NASH) (1-5). While mild fibrosis remains largely asymptomatic, its progression toward cirrhosis, i.e., replacement of functional parenchyma by scar tissue accompanied by severe architectural and vascular distortion, is the major cause of liver-related morbidity and mortality. Clinical sequelae of cirrhosis are (a) liver synthetic (functional) failure, including failing hemostatic, nitrogen handling, and detoxification systems; (b) portal hypertension with consequent formation of ascites and bleeding esophageal or gastric varices; (c) a high susceptibility to infection; and (d) a high risk to develop hepatocellular carcinoma (HCC) (2). Preventive measures, such as antiviral regimens for hepatitis B or C, are already decreasing the burden of viral cirrhosis and HCC, but other causes, such as NASH (which is linked to obesity and type 2 diabetes) are taking center stage. Moreover, numerous patients present initially in the clinic with advanced fibrosis or cirrhosis, which are largely irreversible. Therefore, antifibrotics that prevent progression toward cirrhosis or induce regression of advanced fibrosis and cirrhosis are urgently needed (6-9).

\section{Liver fibrosis progression and reversal}

Research has delineated key mechanisms and cells that determine fibrosis progression (fibrogenesis) and regression (fibrolysis) (1-19). Notably, liver fibrosis has much in common with fibrosis of other organs, such as lungs and kidneys, leading to a crossfertilization of research across organ boundaries. The structural components of the fibrotic ECM, the growth factors, cytokines, chemokines, and proteases, as well as central signaling cascades implicated in fibrogenesis and fibrolysis, are nearly identical in these different tissues (18, 20-22). Importantly, fibrosis is no longer considered static, but the result of a continuous remodeling process. Nonetheless, in contrast to kidneys and lungs, the liver has an extraordinary capacity to regenerate, even in advanced fibrosis.

Conflict of interest: The authors have declared that no conflict of interest exists. Citation for this article: J Clin Invest. 2013;123(5):1887-1901. doi:10.1172/JCI66028.
Fibrosis is intimately linked to wound healing, serving to prevent tissues from disassembly during inflammation, apoptosis, necrosis, and release of lytic enzymes. Fibrosis usually reverses within days to a few weeks following the resolution of tissue damage, as demonstrated in less advanced rodent and human liver fibrosis $(2,8,9,23-25)$. However, the longer the damage persists, often at a low level, the more ECM is deposited. This chronic damage results in increasingly acellular scar tissue and a steep decline of potential reversibility, even after elimination of causative triggers $(26,27)$. Inefficient fibrolysis is due to several factors: (a) lack of cues for ordered cell repopulation and regeneration due to an atypical ECM and the loss of appropriate cellular context, (b) advanced vascular remodeling with architectural distortion, (c) extensive crosslinking of ECM components such as fibrillar collagen that make proteolytic removal difficult, and (d) the disappearance of cellular elements that digest the scar tissue. Here we discuss the cellular and molecular pathways that promote fibrosis progression and highlight current clinical trials as well as improved methods of monitoring fibrosis.

\section{Cellular targets and multicellular fibrogenic units}

Activated myofibroblasts, representing a spectrum of similar ECM-producing cells that mainly derive from hepatic stellate cells and portal fibroblasts, are the major producers of the fibrotic ECM and the most downstream cellular effectors of liver fibrosis (Figure 1). Very few hepatic myofibroblasts in fibrosis stem from BM-derived fibrocytes (12). Moreover, complete epithelial-mesenchymal transition (EMT) of hepatocytes and bile duct epithelia to myofibroblasts may be a rare event - while an "incomplete" EMT of these cells with acquisition of a fibrogenic phenotype is common (28). Myofibroblasts and their products are primary targets for antifibrotic therapies, which in principle would address all types of fibrosis, including advanced fibrosis.

Importantly, additional cellular elements that are either upstream of the myofibroblasts or tightly linked to fibrogenic activation within cellular units may provide a basis for complementary and more disease-specific antifibrotic approaches. A combination therapy approach may be more effective, given that crosstalk between different cell types generally underlies fibrogenic activation. Conceptually, three major multicellular functional units can be defined according to their constituent cell types: (a) perisinusoidal/vascular - pericytes, i.e., hepatic stellate 


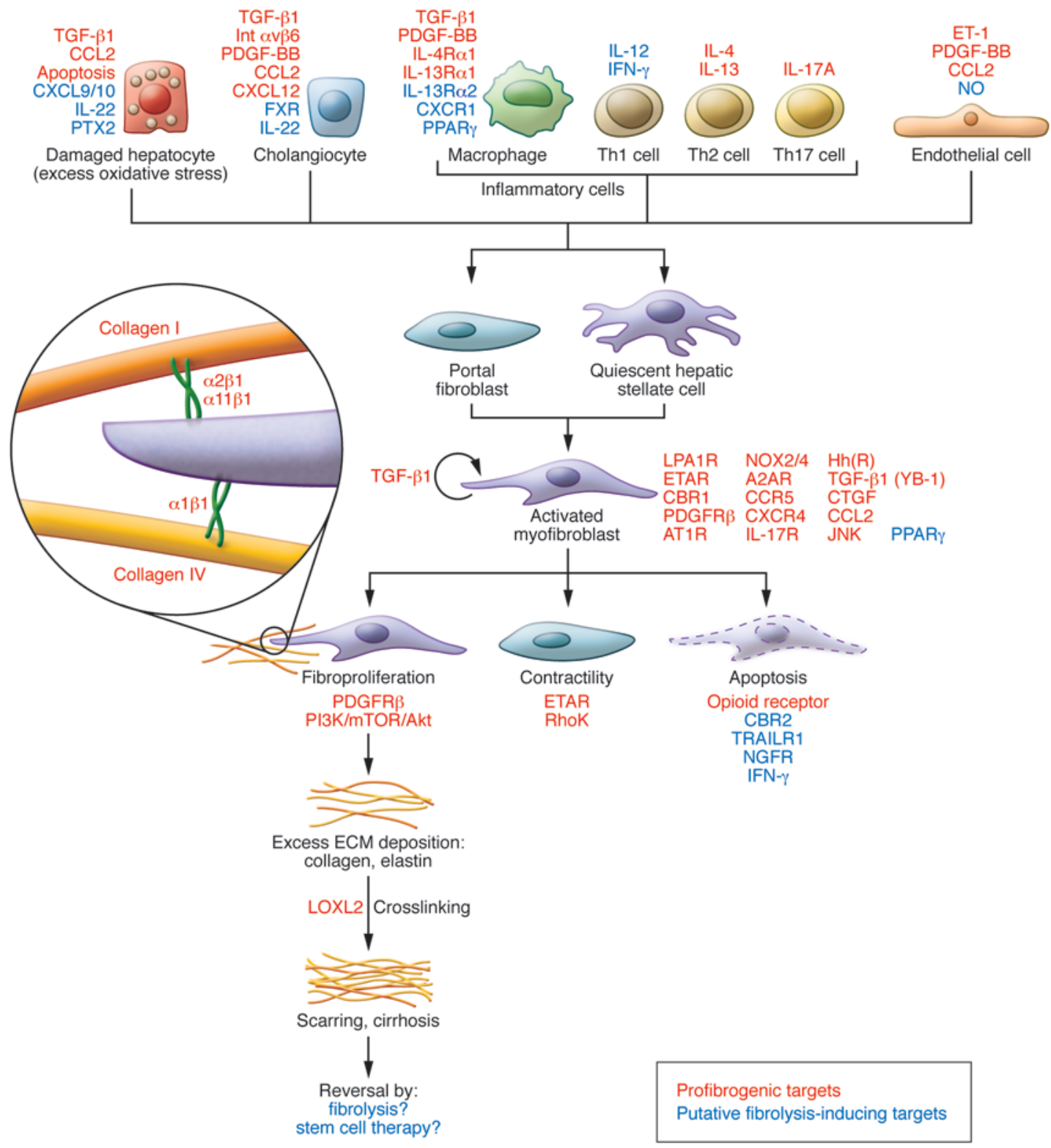

Figure 1

Myofibroblasts and their fibrogenic activation. Cells and major factors upstream of quiescent portal fibroblasts and hepatic stellate cells that induce transformation to fibrogenic myofibroblasts. This schematic highlights several major targets to treat liver fibrosis. Notably, the ECM itself can serve as modulator of fibrogenesis and fibrolysis. Thus collagen fibrils become crosslinked by LOXL2, which contributes to the reduced reversibility of advanced fibrosis, and collagen-binding ECM receptors (especially the integrins $\alpha 1 \beta 1, \alpha 2 \beta 1$, and $\alpha 11 \beta 1$ ) confer signals of stress or stress relaxation that either maintain fibrogenic activation or induce fibrolytic activity of the myofibroblasts. Additional minor contributors to fibrogenic activation are not shown here (see text for details). A2AR, adenosine 2A receptor; AT1R, angiotensin 1 receptor; CBR1, cannabinoid receptor 1; ET-1, endothelin-1; ETAR, endothelin A receptor; FXR, farnesoid X receptor; Hh(R), hedgehog (receptor); Int, integrin; LPA1R, lysophosphatidic acid receptor 1; NGFR, nerve growth factor receptor; PTX2, pentraxin 2; TRAILR, TNF-related apoptosis-inducing ligand receptor; YB-1, Y-box binding protein.

cells, liver sinusoidal endothelial cells (LSECs), macrophages/ Kupffer cells, and hepatocytes; (b) stromal inflammatory myofibroblasts, $\mathrm{T}$ cells, and macrophages; and (c) portal/periportal - cholangiocytes/ductular cells, portal fibroblasts, and various inflammatory cells (ref. 8 and Figure 2, A-C). Altered interactions within these functional units give rise to the major multicellular fibrogenic pathways.

\section{Fibrogenic effectors}

Activated myofibroblasts. Myofibroblasts that derive from both activated hepatic stellate cells and portal fibroblasts are the primary producers of scar tissue $(1,2,6-22,29)$. Notably, myofibroblasts are essential for organ integrity, and their elimination promotes tissue necrosis and inflammation (30). Moreover, myofibroblasts can also contribute to fibrosis regression via release of ECM- 
A

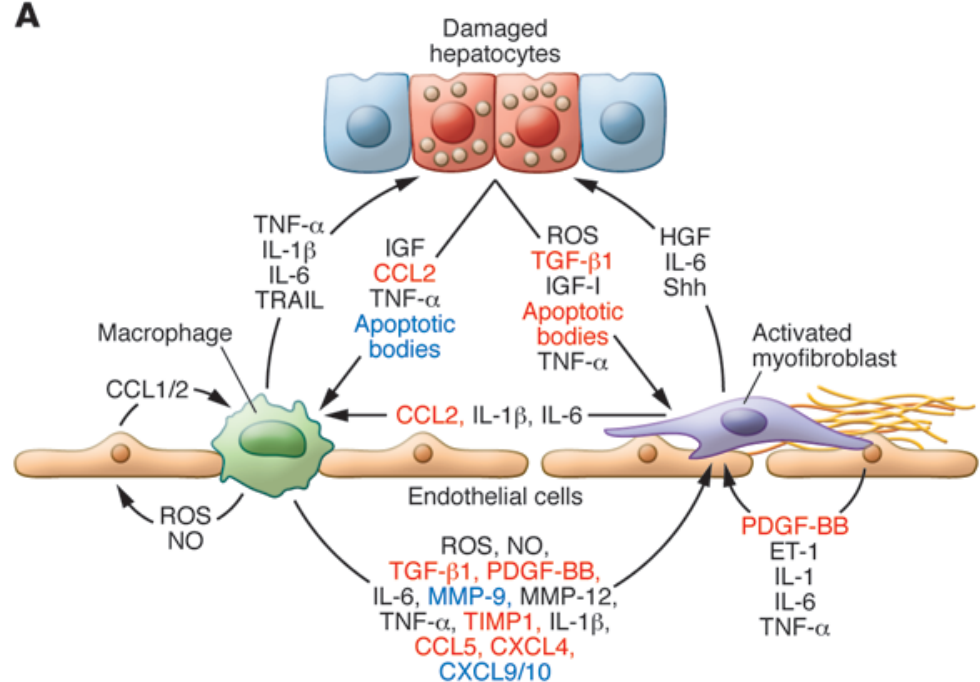

B

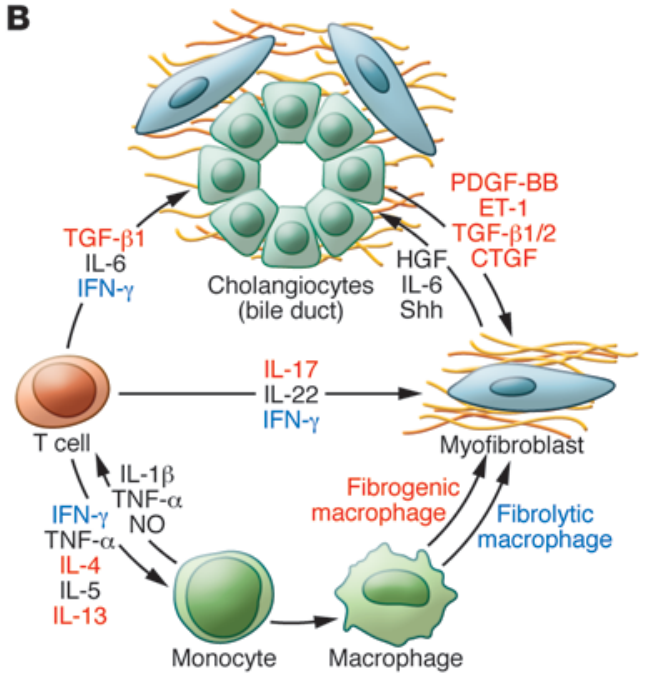

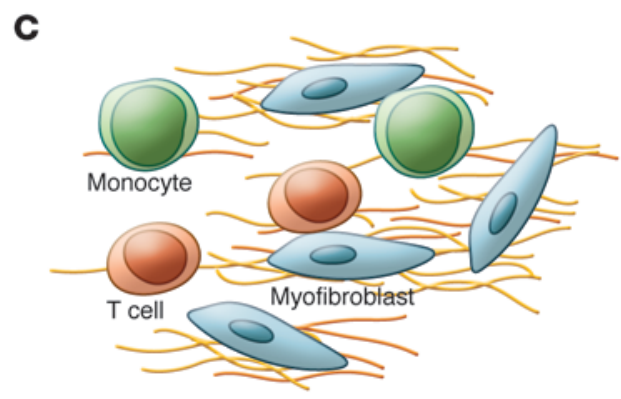

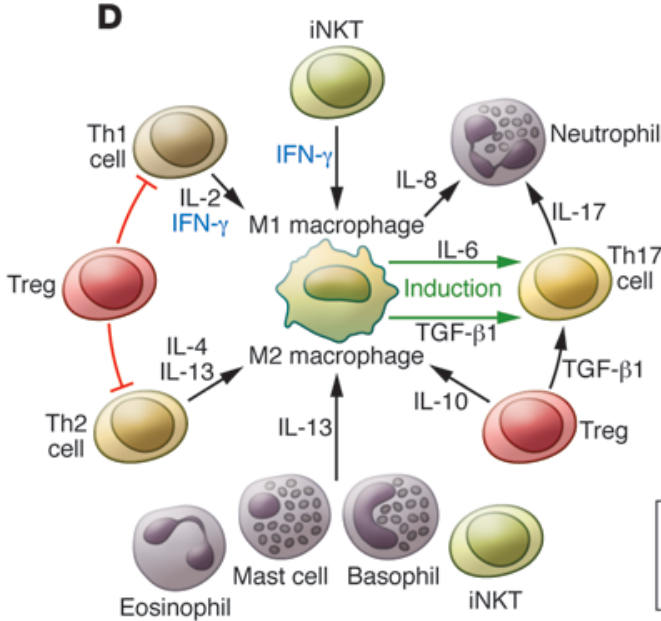

Profibrogenic targets

Putative fibrolysis-inducing targets

Figure 2

Multicellular context of fibrogenesis and fibrolysis. Shown are the postulated major cellular functional units and secreted factors that should be addressed in their complexity when designing effective antifibrotic strategies. (A) Vascular unit. (B) Biliary unit. (C) Inflammatory unit. (D) Cells and factors that affect macrophage polarization. Macrophages (and monocytes as macrophage precursors) are major modulators of inflammation and tissue remodeling. Cells and factors that induce either M1 or M2 polarization are also linked to the generation of fibrogenic Th17 cells and neutrophil recruitment. See text for details. B and C highlight factors not shown in A and B, respectively. Baso, basophil; EO, eosinophil; Mast, mast cell; PMN, polymorphonuclear neutrophil; TIMP, tissue inhibitor of metalloproteinases.

degrading proteases, when confronted with favorable (e.g., ECMderived and integrin receptor-mediated) stimuli, in a process called stress relaxation. Stress relaxation is the basis for limiting ECM deposition once the wound is closed: the activated myofibroblasts contract on the accumulated loose collagen matrix, which triggers release of ECM-degrading proteases, mainly MMPs $(31,32)$. Consequently, treatment strategies should not eliminate myofibroblasts, but rather dampen their fibrogenic activation, confer signals of stress relaxation, and induce fibrolytic enzymes. Accordingly, two rodent studies demonstrated that approximately $50 \%$ of activated hepatic stellate cells/myofibroblasts undergo apoptosis during fibrosis reversal, whereas the rest revert to a quiescent phenotype $(33,34)$. Quiescence can be induced by inhibition of certain fibroblast integrins, cellular receptors that confer mechanical cues in response to ECM attachment (20) with the potential of converting activated to fibrolytic (myo-)fibroblasts (refs. 31, 32, 35, 36 and Figure 1). Specific integrin inhibitors have been developed for cancer therapy, but need better validation for treatment of fibrosis $(37,38)$. Myofibroblast stress relaxation and resultant amelioration of both fibrogenesis and portal hypertension has been shown in rats by inhibition of Rho kinase, which is downstream of integrin signaling (39).

Several agents that block fibrogenic activation and ECM production by myofibroblasts work well in culture and in some rodent models of liver fibrosis but carry a high risk of unwanted side effects in patients due to a lack of specificity for myofibroblasts. Three major strategies are currently in preclinical development to specifically target the pathogenic function of activated myofibroblasts. First, therapies may address fibrosis-relevant pathways that are upregulated in these myofibroblasts, such as procollagen type I 

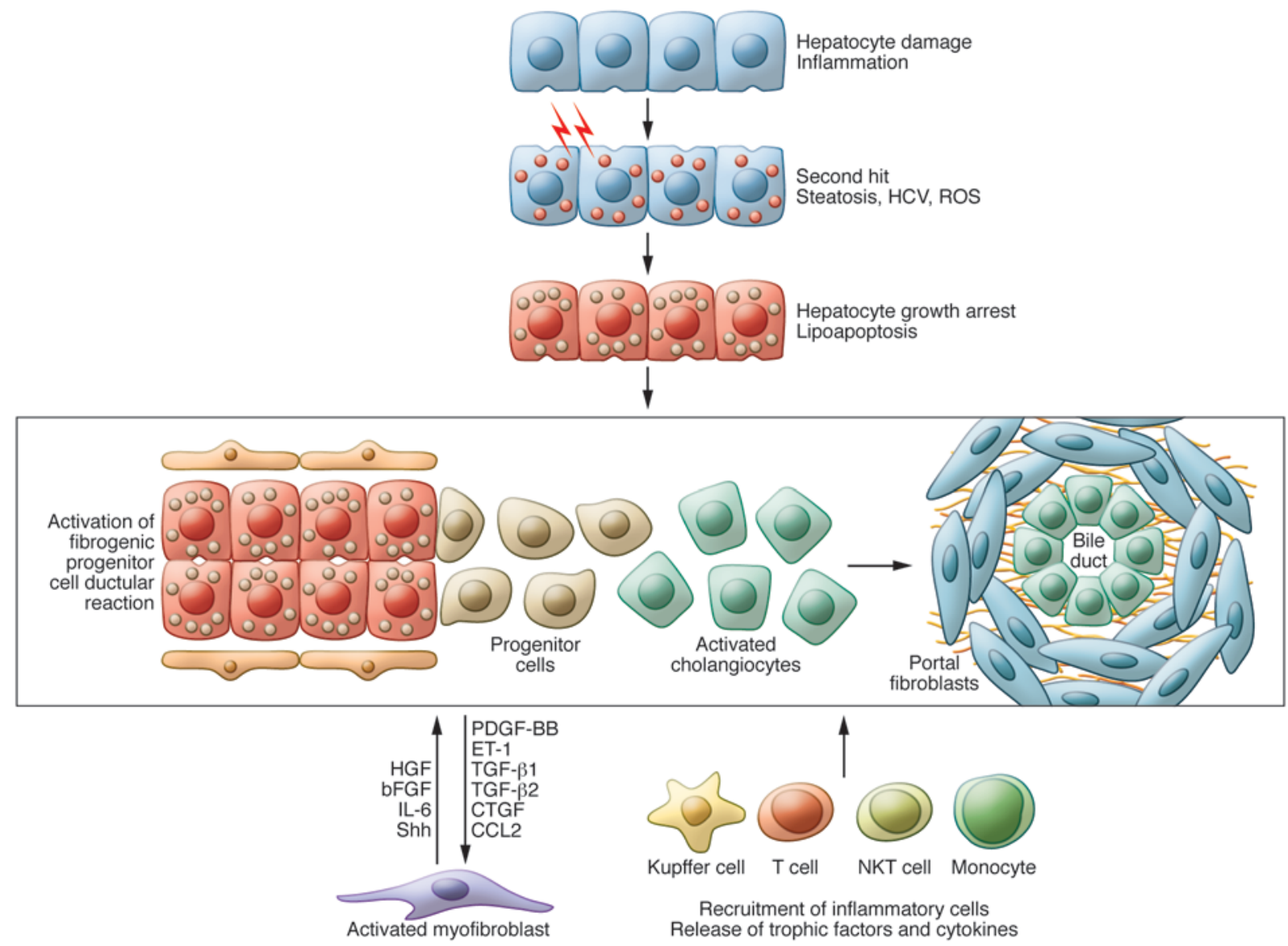

Figure 3

Activated cholangiocytes as drivers of fibrosis progression. Activated cholangiocytes are related, if not identical, to biliary progenitor cells. These cells proliferate in active biliary diseases and during massive hepatocyte growth arrest or apoptosis, as in severe NASH, ASH, or viral hepatitis. Biliary progenitor cells are regularly found in more advanced fibrosis (especially Metavir stage F2 or higher). They replicate ductal plate formation by induction of a portal fibrotic matrix via secretion of profibrogenic factors and recruitment and activation of myofibroblasts, and also Kupffer cells and monocytes and other inflammatory cells like T and NKT cells. The recruited myofibroblasts (and the inflammatory cells) secrete factors and ECM components that maintain these fibrogenic units and support their differentiation into more mature biliary structures that are embedded in a collagen-rich ECM.

or other key structural components of the ECM, or block cellular receptors for ECM components and growth factors/chemokines that are upregulated upon fibrogenic activation. Current blockers of collagen synthesis have unwanted off-target effects, but inhibition of upstream fibrogenic signaling, e.g., PDGFR $\beta$, a strong myofibroblast mitogen, with the tyrosine kinase inhibitor imatinib or a more specific PDGFR $\beta$-blocking antibody retarded early but not advanced liver fibrogenesis $(40,41)$.

A second approach to targeting activated myofibroblasts is to employ refined siRNA delivery techniques, such as liposomal formulations that intrinsically accumulate in liver due to their size, shape, and surface charge, and that deliver cargo to myofibroblasts as well as other liver cell types $(42,43)$. For example, biliary and parenchymal liver fibrosis was significantly mitigated in mice treated with liposomes loaded with procollagen $\alpha 1$ (I) siRNA (44). Finally, the use of ligands specific to receptors on activated myofibroblasts can target drugs or siRNA, thus increasing efficacy and minimizing detrimental off-target effects. Examples supporting this approach in vivo include delivery of IFN via a cyclic PDGFR $\beta$ - binding peptide, of a PDGFR $\beta$-specific kinase inhibitor via mannose-6-phosphate (which addresses the IGF-II receptor), and of Hsp47 (which is involved in collagen processing) via vitamin Acoupled liposomes (45-48). Although these therapies would largely need to be given parenterally, such application can be justified in situations in which treatment is likely to be highly effective, e.g., for reversing advanced fibrosis. Moreover, modifications of delivery systems such as pegylation (49) can be used to increase halflives, permitting once-weekly or once-monthly dosing.

Damaged hepatocytes. Ongoing hepatocyte apoptosis or necroptosis, as occurs predominantly in liver diseases characterized by enhanced oxidative and endoplasmic reticulum stress, lysosomal activation, and mitochondrial damage (ASH, NASH), is a strong trigger of fibrogenesis $(16,50)$. Phagocytosis of apoptotic hepatocytes by myofibroblasts triggers their fibrogenic activation via NADPH oxidase 2 (NOX2) (51) and the JAK/STAT and PI3K/ Akt pathways (52). Notably, inhibition of hepatocyte apoptosis by a pan-caspase inhibitor or an antagonist of cathepsin B (a lysosomal trigger of apoptosis) ameliorated (biliary) fibrosis in mice 
$(53,54)$. On the other hand, as mentioned below, engulfment of apoptotic hepatocytes and biliary cells by macrophages can induce their fibrolytic activation.

Biliary progenitors. The hallmark of biliary fibrosis is the proliferation of biliary progenitor cells (activated cholangiocytes) that tend to form small clusters or usually nonfunctional bile ductular structures, termed ductular reaction. These cells replicate early developmental programs of ductal plate formation, which includes secretion of several factors that attract and activate hepatic stellate cells/myofibroblasts to proliferate and deposit ECM. This biliary progenitor response is amplified by the surrounding myofibroblasts, but also by inflammatory cells that release molecules that sustain ductular cell viability and proliferation (Figure 3). With the exception of infant fibrosis (biliary atresia, Caroli's disease, congenital hepatic fibrosis) and adult primary biliary cirrhosis (PBC), primary sclerosing cholangitis (PSC), and secondary biliary fibrosis, all liver diseases of other etiologies, once advanced, develop into a portal fibrosis with proliferation of biliary progenitors, especially when excessive hepatocyte apoptosis forces the stem cell niche to produce biliary progenitors. These biliary progenitors are more resistant to enhanced oxidative stress and hepatocyte death, such as induced by ASH, NASH, or severe post-transplant hepatitis C (55-60). Drugs aimed at the biliary fibrogenic progenitors are effective antifibrotic agents in rodent biliary and advanced non-biliary fibrosis. Examples are antagonists to the biliary progenitor-specific integrin $\alpha v \beta 6$ (a receptor for fibronectin and tenascin-C, and an activator of latent TGF- $\beta 1$ ) (61-63) or inhibition of the hedgehog pathway, which is primarily upregulated in biliary fibrogenesis and in carcinogenesis $(56-59,64,65)$. Notably, inhibition of hedgehog signaling suppressed biliary fibrosis and even reversed hepatocellular cancer in phospholipid flippase (Mdr2) knockout mice (65).

LSECs. Hepatic (neo-)vascularization with LSEC activation and proliferation is tightly associated with perisinusoidal fibrosis (capillarization of the sinusoids) (Figure 1 and Figure 2A). During perisinusoidal fibrosis, activated LSECs contribute to ECM production (including basement membrane components, fibronectin, and interstitial collagen type I), produce cytokines (e.g., TGF- $\beta 1$ and PDGF-BB) that activate hepatic stellate cells, and secrete factors (e.g., endothelin-1) that contribute to intrahepatic vasoconstriction, which exacerbates portal hypertension in cirrhosis. Conversely, myofibroblasts activate LSEC via secretion of angiogenic factors such as VEGF and angiopoietin-1 (66). Antiangiogenic therapies have mitigated experimental liver fibrosis, mostly in models with a prominent sinusoidal component. However, antifibrotic effects were evident with polykinase inhibitors such as sunitinib and sorafenib that, apart from angiogenic VEGF or FGF receptors on LSECs, also target numerous other cells and kinases involved in proliferation, ECM turnover, and immune regulation $(67,68)$. This lack of specificity may explain the finding that treatment with anti-VEGF antibody and an antagonist to integrin $\alpha v \beta 3$, therapies that inhibit LSEC proliferation (but also affect the proliferation of endothelia of larger vessels) may worsen advanced biliary, perisinusoidal, and interstitial kidney fibrosis (69-71). Moreover, specific inhibition of VEGF mitigates biliary fibrosis progression but retards fibrosis reversal after jejunoileal anastomosis (72). Therefore, as with many other therapies, the antifibrotic efficacy of antiangiogenic therapies is highly context dependent.

T cells. CD $4^{+} \mathrm{T}$ cells with a Th2 polarization, which are prevalent in allergies, asthma, or parasite infections, promote fibrogenesis in the liver, lungs, or kidneys (18, 73-75). Th2 cells produce IL-4 and IL-13, which stimulate the differentiation of potentially fibrogenic myeloid cells and (alternatively) activated (M2) macrophages (refs. 73, 76, and Figure 2D). Thus rodents with Th2-dominant $\mathrm{T}$ cell infiltration (e.g., in experimental schistosomiasis or in experimental models skewed toward Th2; ref. 77) display rapid fibrosis progression, whereas $\mathrm{CD}^{+}$Th1 cells have an antifibrotic effect (78). Accordingly, patients dually infected with HCV and Schistosoma show a 6-fold faster liver fibrosis progression than matched HCVmonoinfected patients (79).

Th17 cells are clear drivers of fibrosis in multiple tissues $(80,81)$. Th17 cells are induced by a special inflammatory environment, including the cytokines TGF- $\beta 1$ and IL- 6 . Th17 cells secrete IL-17A, which drives fibrogenesis directly in myofibroblasts and indirectly via stimulation of TGF- $\beta 1$ release from inflammatory cells $(80,82)$.

Regulatory $\mathrm{T}$ cells appear to either favor or inhibit fibrogenesis, again in a context-dependent manner. Subsets produce various amounts of the immunosuppressive cytokines IL-10 (potentially antifibrotic) and TGF- $\beta 1$ (profibrotic). In most settings of chronic inflammation, TGF- $\beta 1$ prevails.

NK and NKT cells are enriched in the liver and belong to the innate (NK) immune system or the interface between the innate and adaptive (NKT) immune system. In rodent models of liver fibrosis, NK cells repress fibrosis in two ways: (a) by killing earlyactivated or senescent hepatic stellate cells/myofibroblasts that express NK cell ligands and (b) via production of (antifibrotic) IFN (83). In rodent studies, the effect of invariant NKT (iNKT) cells on liver fibrosis is controversial and modest. At best, iNKTs attenuate early but not late toxin-induced fibrogenesis (84), whereas (variable) NKTs worsened fibrosis in the methionine- and choline-deficient diet NASH model (59). Similar to NK cells, beneficial activity may be explained by killing of hepatic stellate cells/myofibroblasts and IFN secretion, but subsets of iNKT cells can also produce profibrotic IL-13. Notably, iNKT cells protected against diet-induced obesity, insulin resistance, and NASH (85), making them a potential therapeutic target for this common cause of liver fibrosis.

Monocytes. Monocytes, which play a key role in inflammation and fibrosis, are also precursors of fibrocytes, macrophages, and dendritic cells and share characteristics with myeloid suppressor cells $(86,87)$. At the interface of innate and adaptive immunity, monocytes help orchestrate adaptive immune responses, with proinflammatory monocytes $\left(\mathrm{Ly} 6 \mathrm{C}^{+} \mathrm{Gr} 1^{+}\right.$in mice; $\mathrm{CD} 14^{+} \mathrm{CD} 16^{+}$ in humans) promoting fibrogenesis $(88,89)$. Chemokines and their receptors are important in monocyte recruitment and activation, representing attractive targets for fibrosis modulation (16, 90). CCL2 and its receptor CCR2 are central to monocyte recruitment to the inflammatory lesion, and their inhibition ameliorates fibrosis progression in rodent models but retards fibrosis reversal (86). Conversely, the chemokine CXCL9 (and CXCL10) prevents pathological angiogenesis and fibrogenesis via activation of their receptor, CX3CR (91-93). Monocytes are also the precursors of circulating fibrocytes, cells that differentiate into collagen-producing fibroblasts and are related to BM mesenchymal stem cells (12). On the other hand, monocytes are the source of fibrolytic CD $133^{+}$ cells that home to liver to induce fibrosis reversal after BM transplantation $(12,17)$. Chemokines and their receptors are important in monocyte recruitment and activation, representing attractive targets for fibrosis modulation.

Macrophages. These resident cells derive from circulating monocytes as precursors (partly replenishing the liver specific Kupffer 


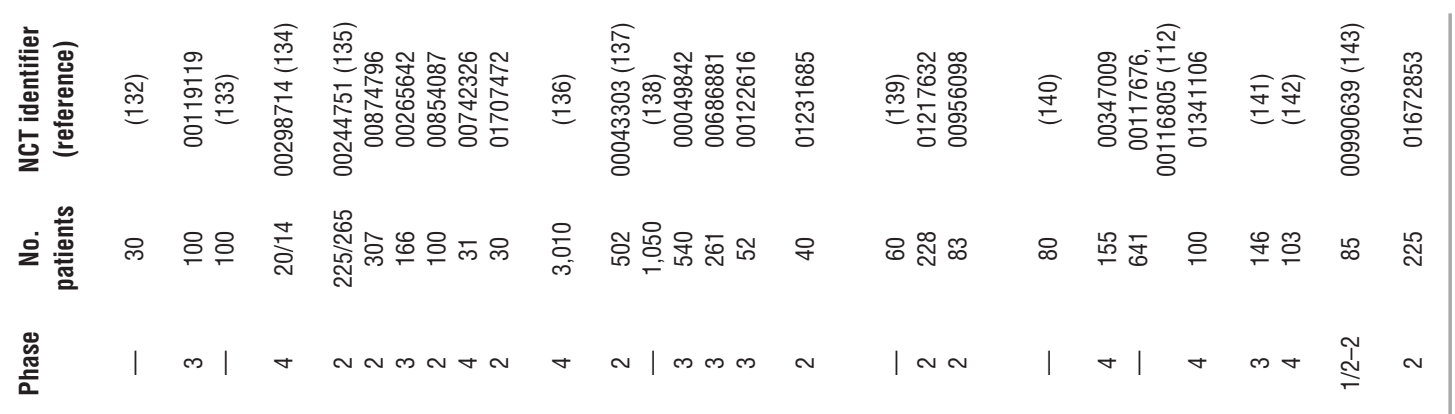

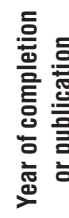

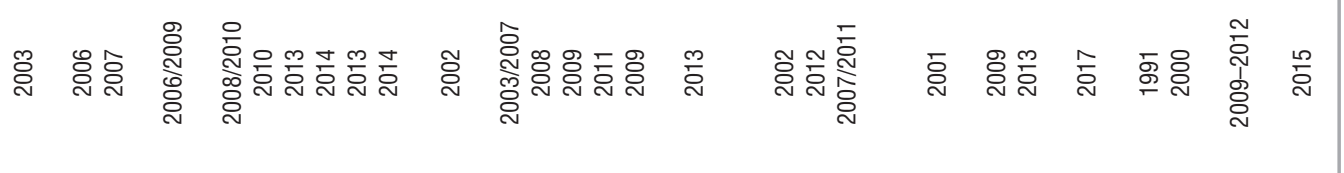

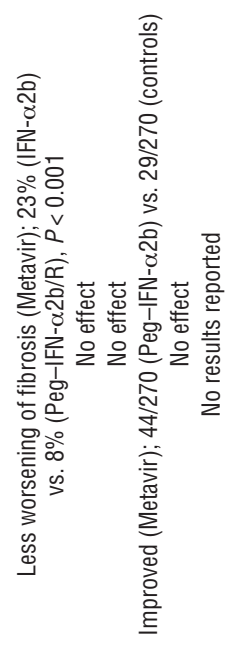

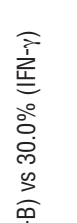

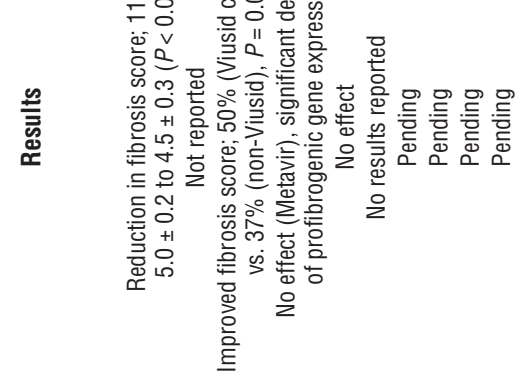

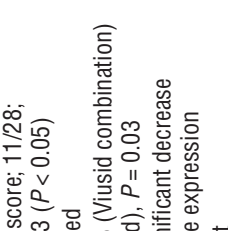

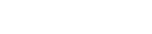

(

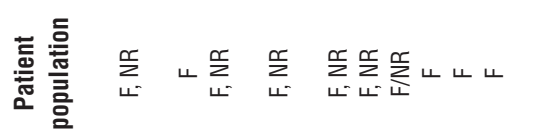

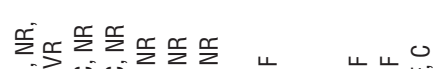

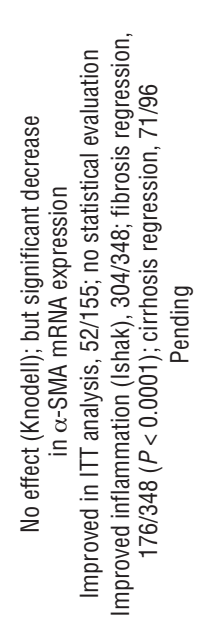

产

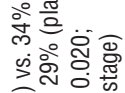

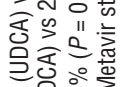

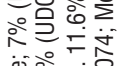

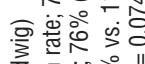

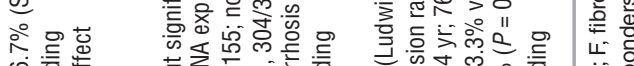

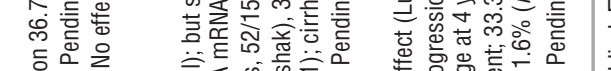

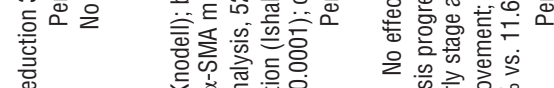

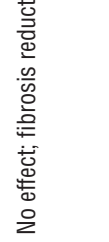

을

혼

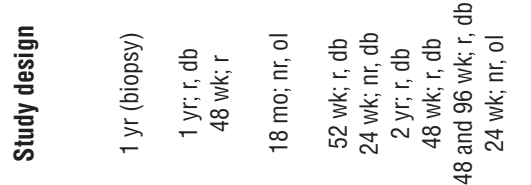

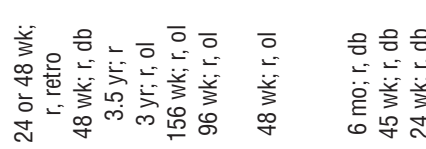

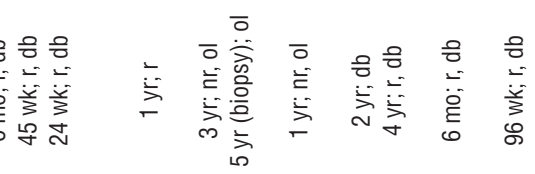

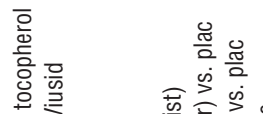

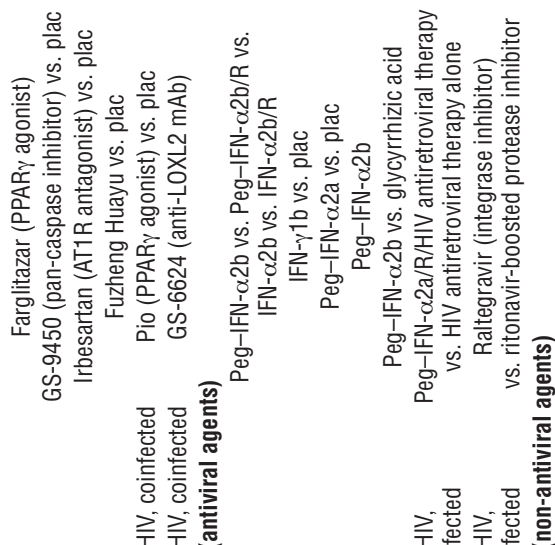

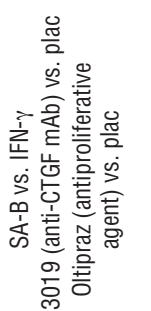

它

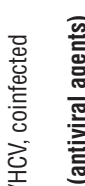

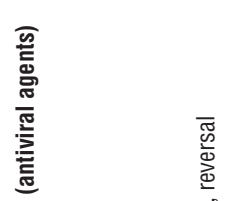

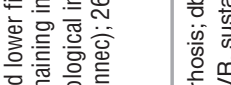

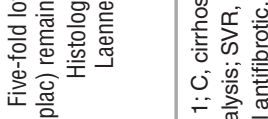

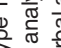

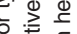

造 잉

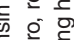

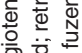

步

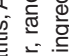




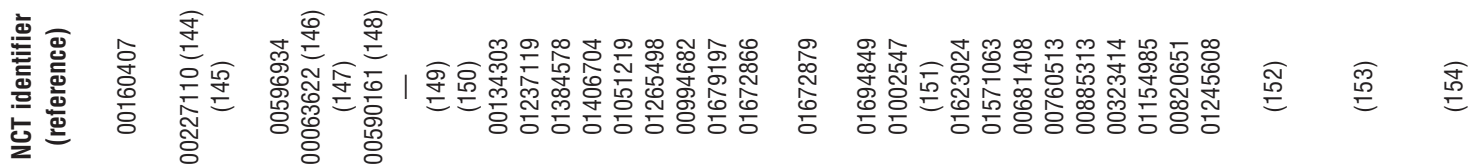

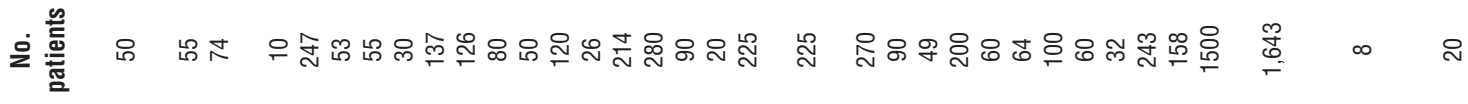

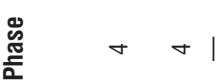

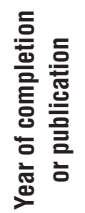

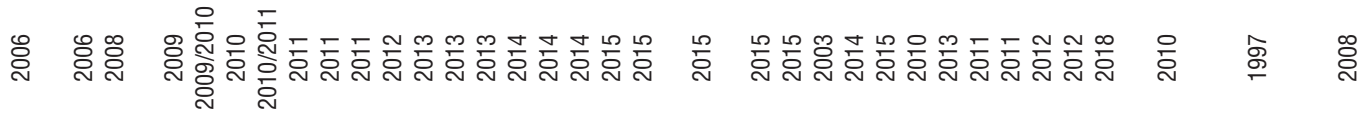

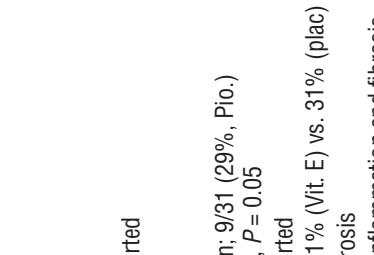

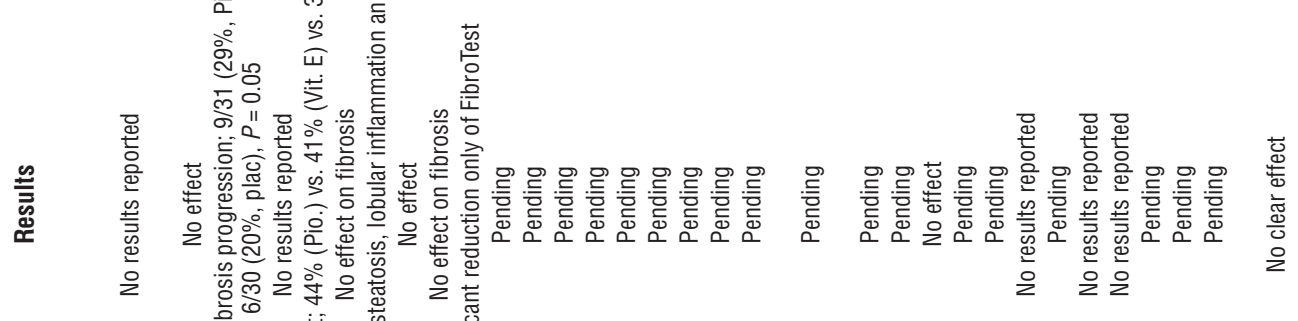
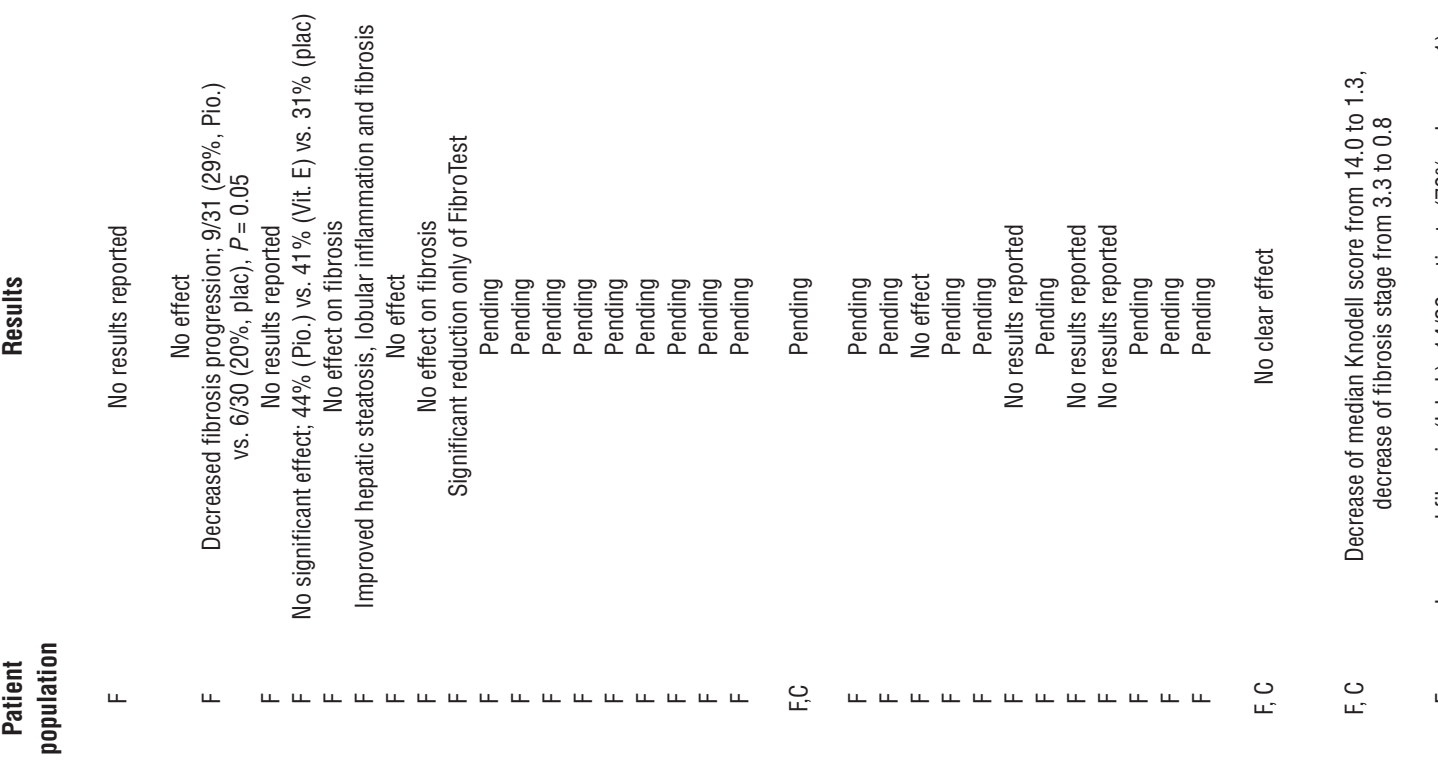

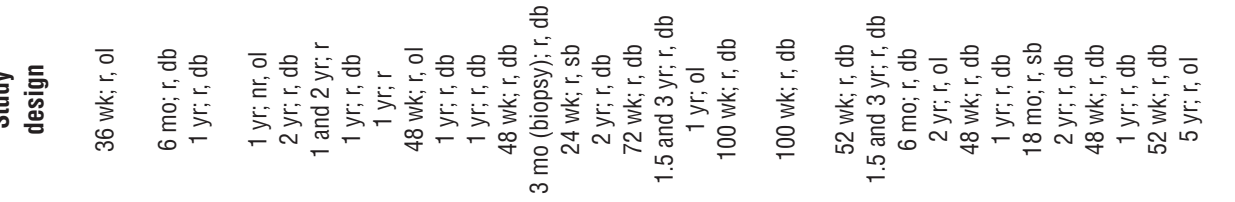
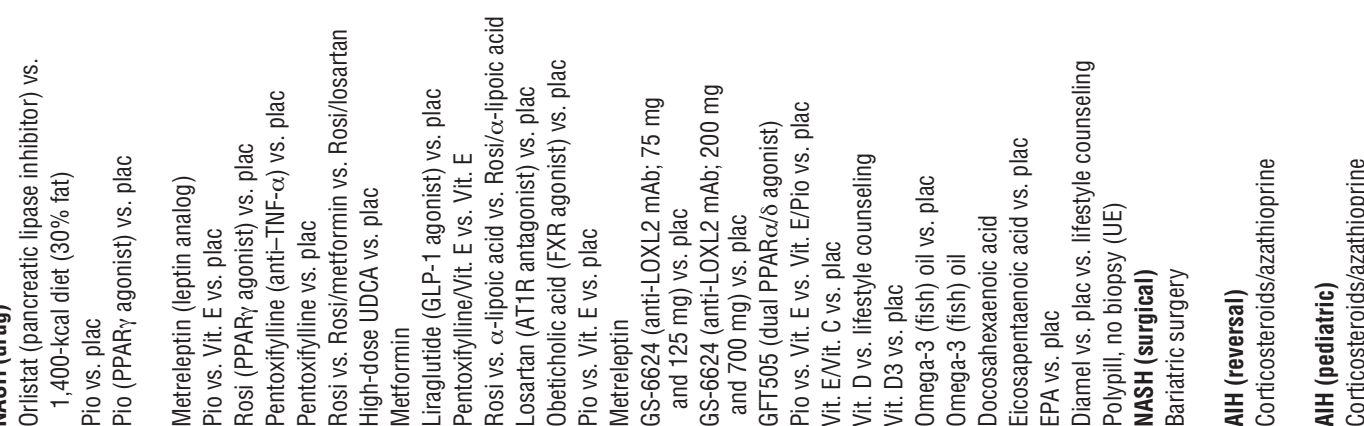

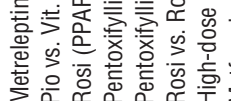

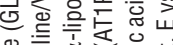


cells). M1 macrophages are induced by IFN or IL-12, while IL-4, IL-13, and GM-CSF induce M2 macrophages. Macrophages appear to be fibrogenic during fibrosis progression and fibrolytic during its reversal, but a detailed functional analysis and assignment to M1 or the various M2 subclasses has remained elusive $(18,23,26)$. While M1 macrophages are activated in immediate defense against pathogens or detrimental cellular debris, M2 macrophages are generally thought to promote wound healing (i.e., fibrogenesis) and immune suppression (e.g., facilitating cancer growth as tumorassociated macrophages) $(18,94,95)$. M2 macrophages respond to IL-4 and IL-13 via IL-4 receptor and IL-13 receptor $\alpha 1$ (with IL-13 receptor $\alpha 2$ serving as negative regulator) and are characterized by unique signal transducers (e.g., Stat6), enzymes (e.g., arginase), or scavenger receptors (e.g., CD206). However, several subtypes of M2 macrophages exist, such as the putatively proinflammatory $\mathrm{M} 2 \mathrm{a}$, and the anti-inflammatory M2b and M2c subtypes, which have ill-defined roles in fibrosis $(20,74)$. A recent study demonstrated that fibrolytic macrophages in liver fibrosis derive from circulating Ly6Chi-expressing monocytes and develop locally into Ly $6 \mathrm{C}^{\mathrm{lo}}$-expressing macrophages with some classical M2 markers and a high expression of fibrolytic MMPs, and this development depends on phagocytic activity (96). Notably, MMP release depends on phagocytosis of apoptotic cells, which is also a driver of biliary fibrosis reversal (23). Given that M1 polarization in liver and adipose tissue enhances insulin resistance and promotes inflammation in NASH, whereas M2 polarization is protective (97), the targeting of macrophage polarization in liver inflammation and fibrosis is an attractive therapeutic option.

\section{Other relevant molecular targets}

Several other molecular targets are of interest, and some have already entered clinical studies. ECM cross-linking, mainly of fibrillar collagen, is largely mediated by lysyl oxidase (LOXL2). LOXL2 likely impedes ECM degradation during fibrosis reversal, and antifibrotic activity has been seen in a small study of CCL4induced liver fibrosis (98). A humanized antibody that blocks LOXL2 activity is currently assessed in the largest clinical study for liver fibrosis (Tables 1 And 2).

TLRs are sensors of bacteria, viruses, and foreign antigens. TLRs are expressed ubiquitously but are prominent on cells of the innate immune system, creating a proinflammatory environment and activating adaptive immunity to promote pathogen elimination. As the major interface between the gut and systemic circulation, liver cells are equipped with a variety of TLRs that are central to both maintaining immune tolerance and initiating inflammation and repair when confronted with (microbial) danger signals (99). A direct link exists between liver fibrosis and bacterial LPS, and activation of its receptor TLR4. LPS enters the portal hepatic circulation in conditions of enhanced intestinal permeability, such as in ASH, NASH, and other intestinal and liver diseases. LPS upregulates chemokine secretion of monocytes and macrophages/ Kupffer cells and downregulates the inhibitory TGF- $\beta 1$ pseudoreceptor Bambi, which cumulatively sensitizes hepatic stellate cells/myofibroblasts to fibrogenic activation $(99,100)$. Prevention of excessive TLR 4 activation or inhibition of TLR 4 are therefore attractive strategies to inhibit fibrogenesis. Currently only the parenteral TLR4 antagonist, eritoran tetrasodium, is being studied for the treatment of sepsis (101). Other interesting but little explored targets include TLR3, a double-stranded RNA sensor whose activation by polyI:C attenuates liver fibrosis via activation of NK cells
(102), and TLR9, a receptor for double-stranded bacterial DNA that enhances fibrogenic immune activation via release of CCL2 (103). In addition, inhibitors of broadly expressed chemokine systems other than CCR2/CCL2, mainly CXCL4 and CCL5 (and their receptors CXCR4 and CCR5, respectively) on myofibroblasts, $\mathrm{T}$ cells, and macrophages, have been shown to attenuate liver fibrosis (104-106). Furthermore, the recent explosion of data related to microRNAs (miRs) has uncovered miRs that inhibit (miR-29b) or promote fibrogenesis (miR-199, miR-200, and others) (107-109). While these miRs appear to have some specificity for myofibroblasts, their efficient in vivo delivery poses a problem.

TGF- $\beta$ and, to a lesser degree, its downstream mediator, connective tissue growth factor (CTGF), are potent profibrogenic cytokines for hepatic stellate cells/myofibroblasts (1, 2, 6, 9, 10, 18, 19). However, their general and untargeted inhibition poses risks, especially for TGF- $\beta$-neutralizing agents, given that this cytokine is central to cellular differentiation, immune regulation (dampening excessive $T$ cell activation), and regulated wound healing, such as in vascular plaque stabilization in atherosclerosis (110).

\section{Preclinical proof of concept}

Before entering clinical studies, best preclinical proof of antifibrotic activity needs to be obtained in complementary rodent models that reflect different aspects of human liver fibrosis (6). Moreover, drug testing in cultures of precision-cut human liver slices obtained from operations permit a first translation toward the human in vivo system (111).

\section{Combination therapies}

Combination therapies that address liver fibrosis in a multipronged approach hold much promise for future treatment, ideally targeting interactions between cells, soluble mediators, the ECM and its receptors, and/or relevant intracellular signaling. Combinations of targeted antifibrotic agents have yet to be thoroughly tested in preclinical studies. Significant expense and effort will be required to rigorously validate combinations at different doses and in several rodent fibrosis models. However, combinations of specific drugs can be anticipated that interfere with fibrogenesis, induce fibrolysis, or address different cell types.

Clinical development of combination therapies that could guarantee thorough efficacy and low toxicity is only feasible with the advent of improved noninvasive biomarkers and technologies to measure fibrosis, and especially fibrogenesis. Moreover, the necessary personalized approach to the patient with liver fibrosis or cirrhosis will only be possible with such biomarkers, permitting the adjustment of different medications and their dose according to a readily measurable treatment effect.

\section{Testing antifibrotics in clinical trials}

Recent clinical trials with efficient causal therapy have demonstrated reversibility of advanced liver fibrosis. Perhaps the best example is a study of 348 patients with chronic hepatitis B who were treated with the potent antiviral tenofovir (112). After five years, regression of fibrosis was observed in $91 \%$ of patients with significant fibrosis at study entry. Only 12 of 252 patients (5\%) showed fibrosis progression, while 71 of the 96 patients (74\%) with cirrhosis at baseline were no longer cirrhotic at year five. Moreover, all but one of these individuals had at least a two-unit reduction (out of a possible total of six units) in Ishak fibrosis score at year five, a difference that strongly rules out biopsy sampling variability. 


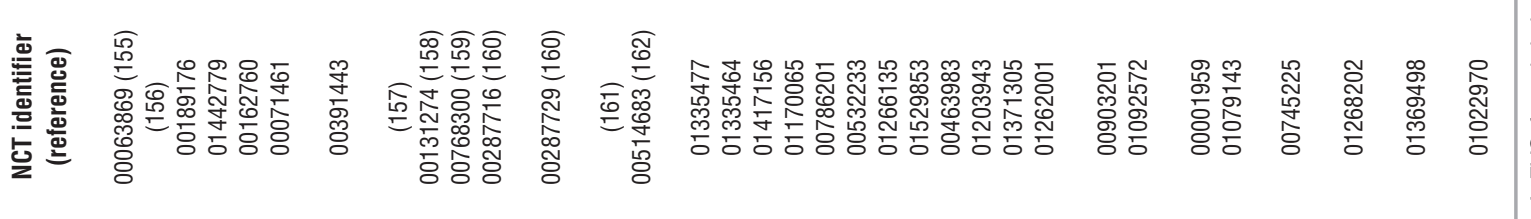

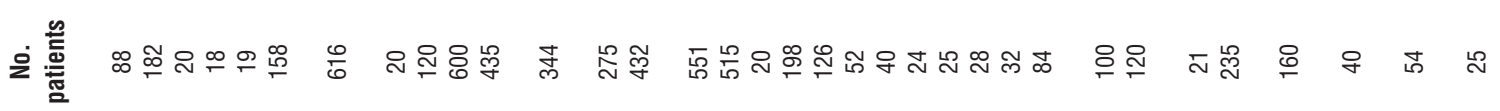

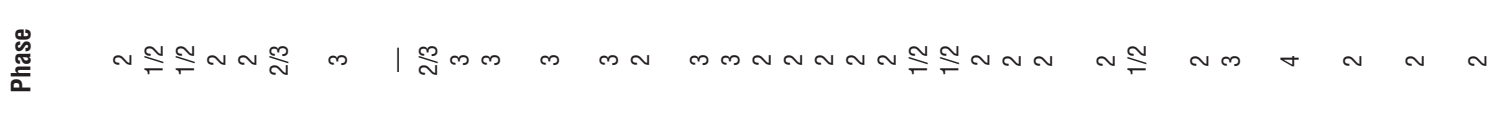

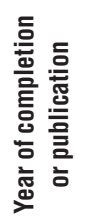

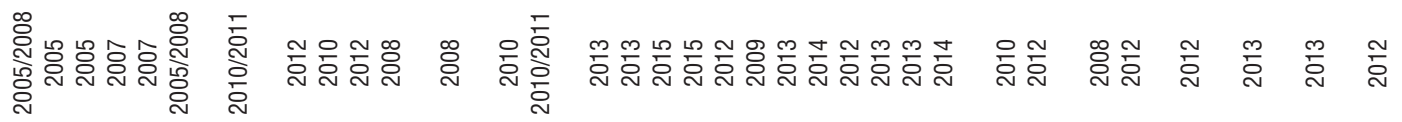
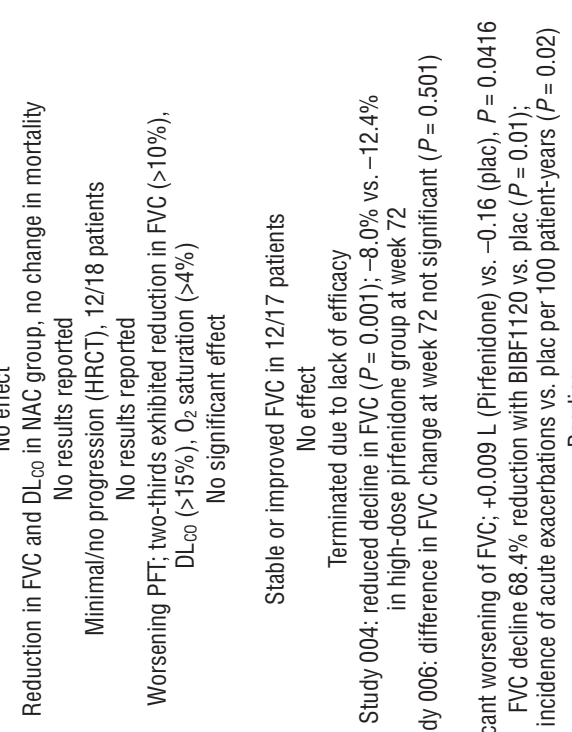

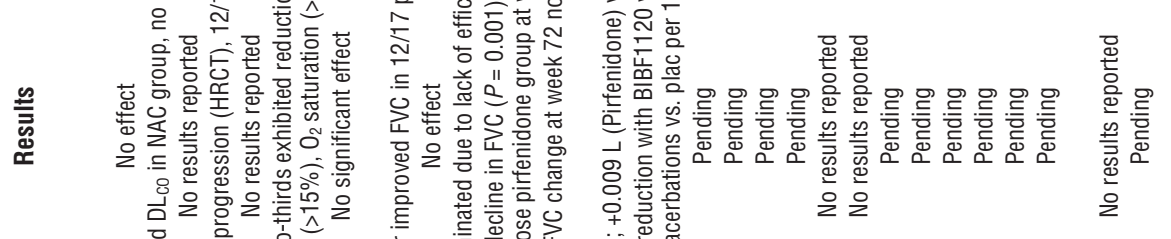<smiles>C1CCCC1</smiles>

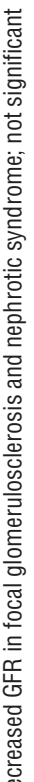

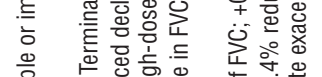

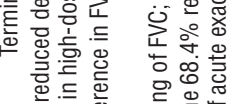

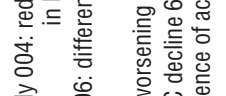

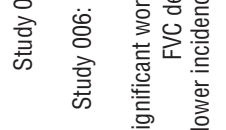

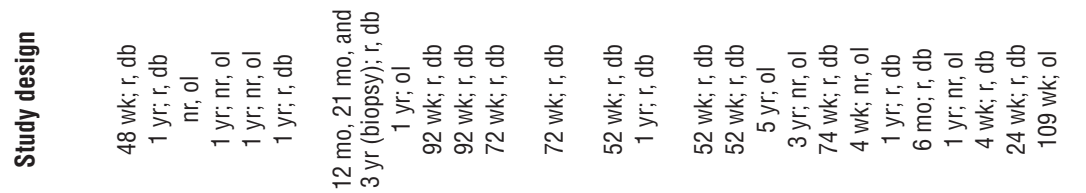

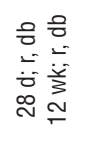

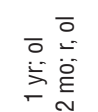

石

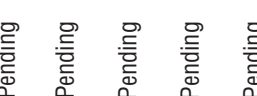

픔

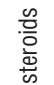

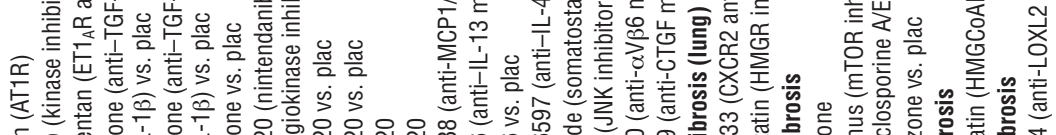




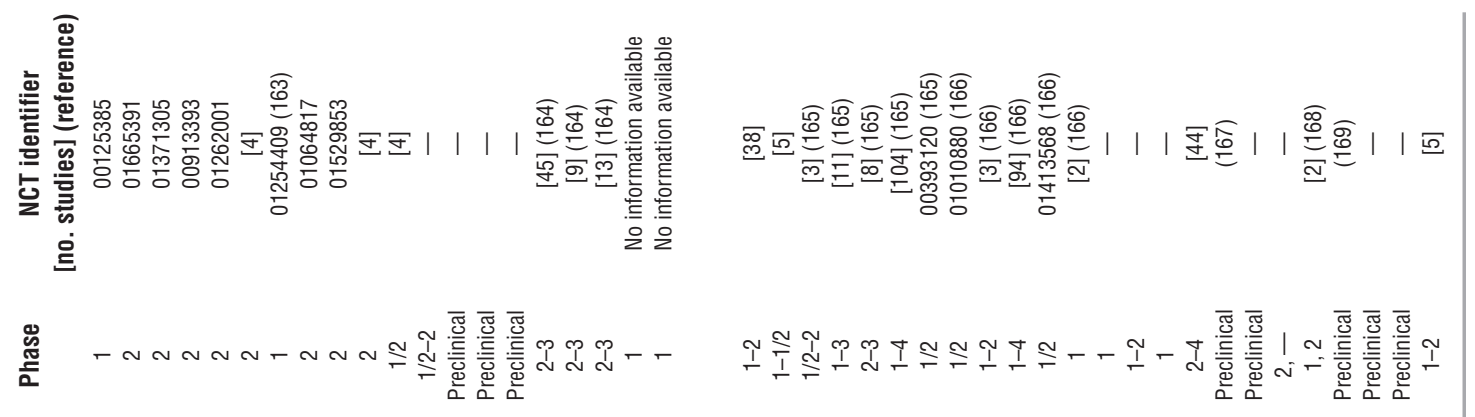

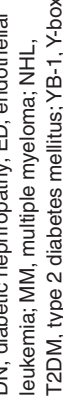

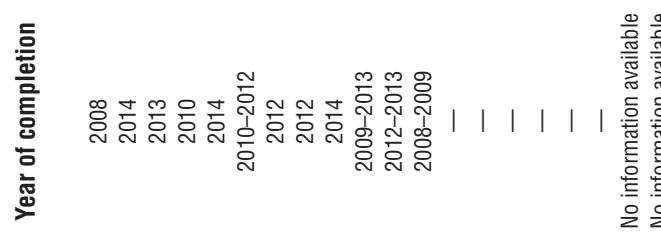

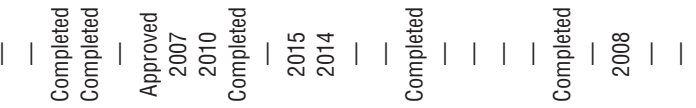

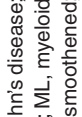

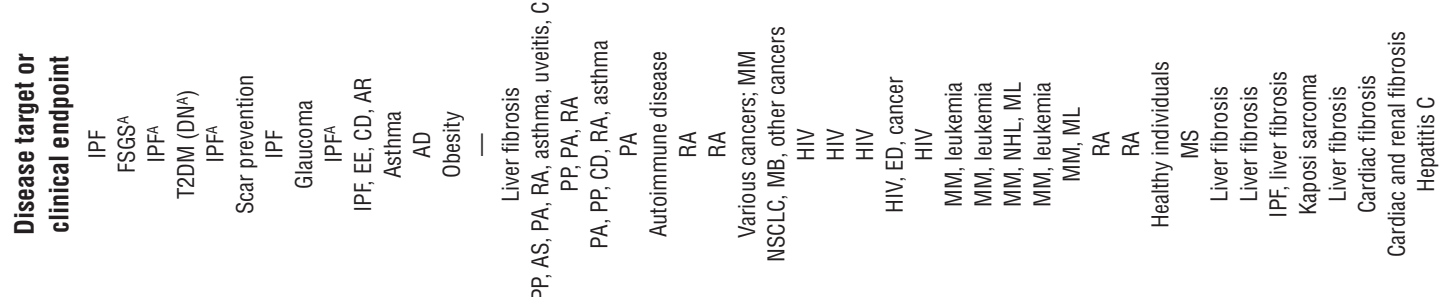

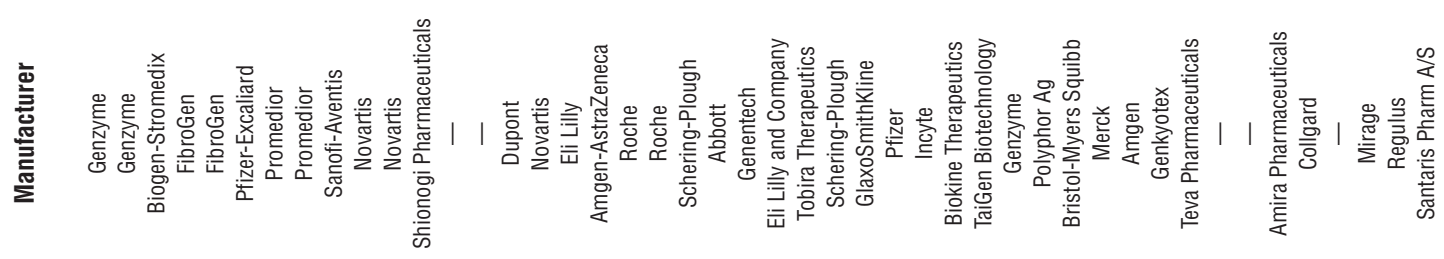

$$
\text { 高 }
$$

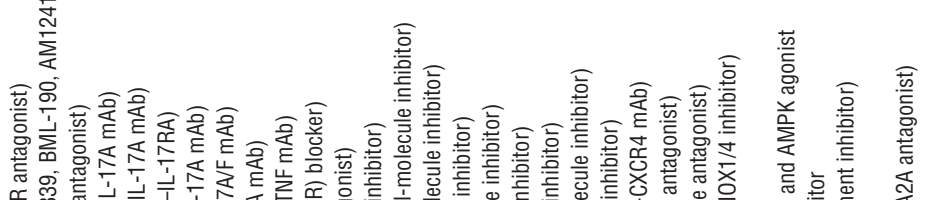

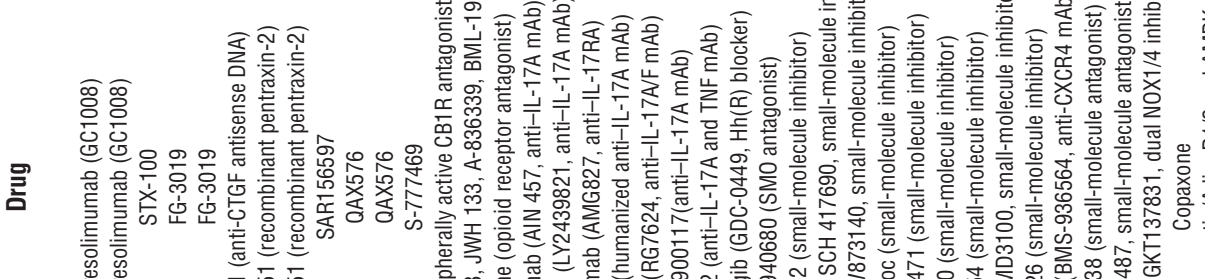

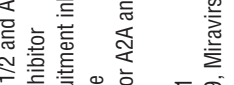

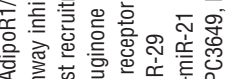

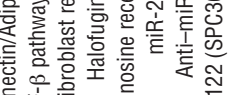

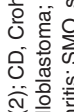
产을

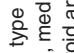
흥

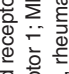

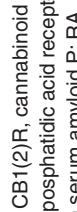


Table 5

Stem cell therapies for liver fibrosis and cirrhosis

\begin{tabular}{|c|c|c|c|c|c|c|}
\hline Cell type & Intervention & $\begin{array}{c}\text { Patient } \\
\text { population }\end{array}$ & Evidence of efficacy & Phase & $\begin{array}{c}\text { No. } \\
\text { patients }\end{array}$ & $\begin{array}{l}\text { NCT identifier } \\
\text { (reference) }\end{array}$ \\
\hline UC-MSC & ol & C & $\begin{array}{l}\text { Improved liver function, MELD, } \\
\text { and reduced ascites }\end{array}$ & - & 45 & (Summarized in ref. 170) \\
\hline UC-MSC & ol & C & $\begin{array}{l}\text { Improved liver function, MELD, } \\
\text { and increased survival }\end{array}$ & - & 43 & (Summarized in ref. 170) \\
\hline BM-MSC & ol & C & Improved liver function and MELD & - & 158 & \\
\hline BMNC & ol & C & Improved ascites and MELD & - & 40 & \\
\hline CD34+ & ol & C & Improved MELD & - & 4 & \\
\hline PBMC from G-CSF & ol & C & $\begin{array}{l}\text { Improved liver function, reduced } \\
\text { Child-Pugh score }\end{array}$ & - & 40 & \\
\hline CD133+ BMSCs & ol & C & Increased liver volume after liver resection & - & 6 & \\
\hline BM-MSC & $r$, ol & C, alcohol & Pending & 2 & 12 & 01741090 \\
\hline BM-MSC & $\mathrm{r}, \mathrm{ol}$ & C, HBV & Pending & 2 & 240 & 01728727 \\
\hline UC-MSC & $\mathrm{r}, \mathrm{ol}$ & C, PBC & Pending & $1 / 2$ & 100 & 01662973 \\
\hline UC-MSC & $\mathrm{r}, \mathrm{sb}$ & C, reversal & Pending & $1 / 2$ & 200 & 01233102 \\
\hline UC-MSC & $\mathrm{r}, \mathrm{ol}$ & $\mathrm{C}$, reversal & Pending & $1 / 2$ & 45 & 01220492 \\
\hline HMB-MSC & $\mathrm{r}, \mathrm{ol}$ & $\mathrm{F} / \mathrm{C}$, reversal & Pending & $1 / 2$ & 50 & 01483248 \\
\hline BM-MNC plus CD133+ & $\mathrm{r}, \mathrm{db}$ & C, reversal & Pending & $1 / 2$ & 30 & 01120925 \\
\hline PBSC & $\mathrm{r}, \mathrm{ol}$ & C, HBV & Pending & $1 / 2$ & 20 & 01728688 \\
\hline $\begin{array}{l}\text { ABMSC plus portal } \\
\text { hypertension surgery }\end{array}$ & $\mathrm{nr}, \mathrm{ol}$ & C & Pending & $2 / 3$ & 50 & 01560845 \\
\hline
\end{tabular}

ABMSC, autologous BM stem cell; BM-MNC, BM mononuclear cell; BM-MSC, BM mesenchymal stem cell; BMNC, blood mononuclear cell; HMBMSC, human menstrual blood-derived mesenchymal stem cell; PBSC, autologous peripheral blood stem cell; UC-MSC, umbilical cord-derived mesenchymal stem cell.

While some additional human studies also suggested antifibrotic activities of tenofovir, others failed to show an effect. Tables 1 and 2 list past and current clinical studies with liver fibrosis as primary endpoint, and Supplemental Table 1 (supplemental material available online with this article; doi:10.1172/JCI66028DS1) shows studies with liver fibrosis as a secondary endpoint. Table 3 highlights clinical studies for other fibrotic diseases that are relevant for liver. Clinical studies targeting major multicellular fibrogenic pathways are shown in Table 4. We also summarize trials employing stem cells, an approach that is attractive in combination with pharmacological therapies (Table 5). Notably, development efforts have largely focused on extracellular targets because intracellular targets are less accessible and tend to lack specificity for the fibrogenic cells.

Optimal selection and stratification of patients. Subjects should be matched according to etiology, age, gender, signs of the metabolic syndrome, medications, and risk factors such as alcohol or tobacco consumption (7-9). Preferably, patients should be in an intermediate fibrosis stage, where dynamic changes of fibrosis are best detectable. Patients with chronic HCV infection following liver transplant are considered a preferred study population because up to $30 \%$ experience an accelerated fibrosis progression to cirrhosis within three to five years (60). Patients should be further stratified according to their genetic risk to progress to cirrhosis $(7,8,60,113)$.

\section{Assessing fibrosis}

Assessment of fibrosis progression is far more difficult for liver than for lungs or kidneys because transaminases do not correlate with fibrosis or fibrogenesis and liver function parameters such as albumin or prothrombin time (protein synthesis) are usually only altered in cirrhosis. Liver biopsy remains the standard for studies with antifibrotics. However, liver biopsy is invasive and risky $(2,6,8,60,114)$ and prone to considerable sampling error, and its interpretation is subject to interobserver variability. Even in well-stratified cohorts, given the usually slow fibrosis progression, conventional fibrosis staging (Metavir, Ishak) may require approximately 200 patients studied over a period of two to three years to detect a $20 \%-30 \%$ difference in fibrosis between treatment groups. However, by including current surrogates of fibrosis progression, it appears feasible to conduct proof-of-concept trials in approximately 100 patients within 12 months or less.

Refined liver biopsy readouts. Predictive value may be improved using dynamic biopsy-derived parameters, such as semiquantification of activated $\alpha$-SMA-positive myofibroblasts and the fibrogenic cytokine TGF- $\beta$ after immunostaining (115), or quantitative PCR quantification of transcripts that are related to fibrogenesis or fibrolysis (116).

Radiological imaging. Conventional and contrast ultrasonography, computerized tomography, and MRI, PET, single-photon emission computerized tomography, and diffusion-weighted MRI cannot differentiate fibrosis stages. However, magnetic resonance texture analysis, which requires sophisticated instrumentation and software, may permit semiquantitative fibrosis assessment $(4,8)$.

Elastography. Ultrasound elastography (UE) and axial radiation force imaging (ARFI) measure hepatic stiffness and elasticity. These techniques sample a 100-fold-larger volume than biopsy and can differentiate mild (Metavir F0-F1) from significant fibrosis (F2-F4) and cirrhosis (F4), with diagnostic accuracies (area under receiver operating characteristics [AUROC] curves) around and above 0.90 , which is considered good $(8,114,116)$. Magnetic resonance elastography assesses the whole liver and may be superior to UE/ARFI, but is not generally available, and studies are 
small $(8,117)$. UE is useful for initial stratification of patients according to fibrosis stage.

Serum fibrosis markers. More than 2,000 studies in the last five years have employed serological markers to assess liver fibrosis, mostly in patients with $\operatorname{HCV}(2,6,8,60,118-121)$. These markers reflect liver function (indirect markers), are related to matrix metabolism (direct markers), or both. The best marker panels show AUROCs around $0.8-0.85$ to differentiate between no/mild fibrosis (Metavir F0-F1) and moderate/severe fibrosis (F2-F4). Fibrosis markers have almost exclusively been validated as predictors of fibrosis stage, while especially the direct parameters may rather reflect the dynamics of fibrogenesis and/or fibrolysis $(2,8)$. Recent studies suggest that certain marker combinations such as the (indirect) Fibrotest (122) and the (direct) enhanced liver fibrosis (ELF) test can predict hard endpoints. Thus ELF was superior to fibrosis stage, the Child-Pugh or the Model for End-Stage Liver Disease (MELD) score, to predict hepatic decompensation or death in long-term, retrospective follow-up studies of patients with advanced chronic HCV or PBC (123-125).

Combination of methods. The combination of serum fibrosis markers with elastography increases diagnostic accuracy, permitting a clear allocation to either no/mild (F0-F1) or significant (F2-F4) fibrosis in $70 \%$ of patients $(8,114)$.

Measurement of portal bypertension and quantitative liver function. The hepatic vein pressure gradient (HVPG) is an excellent predictor of decompensation or death in patients with cirrhosis (126). A non-invasive alternative, the hepatic vein arrival time of an injected ultrasound contrast agent, needs further validation (127).

Tests that measure the metabolic capacity of the liver, such as demethylation of ingested methacetin and quantification of the exhaled metabolite ${ }^{13} \mathrm{CO}_{2}$, correlate inversely with the severity of liver inflammation and fibrosis, and the results of such tests can complement antifibrotic drug trials (128).

Quantitative imaging of liver fibrosis and fibrogenesis. Methods that employ a small molecular ligand for fibrillar collagen, elastin, or a cell-associated molecule coupled to a radio-imaging or MRI agent are in development $(4,8)$. Examples include an elastin-specific MRI probe for imaging of fibrosis (129) and probes for quantifying fibrogenic cells via the cholangiocyte integrin $\alpha v \beta 6$ or the myofibroblast-specific PDGFR $\beta(4,8)$. When improved, such method- ology could serve as a novel gold standard for the assessment of fibrosis/fibrogenesis and permit short-term testing of potential antifibrotics before and after a single dose of the drug.

Novel biomarkers. Apart from ongoing efforts to find and validate better serum markers of fibrosis, fibrogenesis, and fibrolysis $(4,8)$, three methodologies will likely become relevant for antifibrotic drug trials: (a) urinary assessment of proteolytic activities in the fibrotic liver could be monitored after injection of mass-encoded proteasesensitive peptides conjugated to nanoparticles and multiplexed detection of cleavage products by mass spectrometry $(2,130)$; (b) membrane microparticles, which are shed from activated or apoptotic cells, can be quantified in the bloodstream via their cell-specific surface molecules; microparticles represent a novel set of quantitative diagnostic markers to monitor cell-specific activation in liver inflammation and fibrosis $(3,131)$; and (c) circulating miRs that can reflect liver-specific pathology, including hepatocyte differentiation and activation, cancer growth, and liver fibrosis (109).

\section{Conclusions}

We have gained remarkable insight into the cellular and molecular mechanisms of liver fibrosis and reversal, and even reversal of cirrhosis appears feasible in preclinical models. Currently, the field has progressed toward clinical translation. As antifibrotics address mechanisms that are embedded in a complex multicellular network, their efficacy is predicted to be context dependent. Combination therapies hold most promise, but their development and use require a personalized medicine approach that depends on the development and validation of novel noninvasive markers and techniques to quantify liver fibrosis and especially fibrogenesis.

\section{Acknowledgments}

D. Schuppan received funding from the NIH, the European Union, the state of Rhineland-Palatinate, the German Research Foundation, and the German Ministry of Education and Research.

Address correspondence to: Detlef Schuppan, Institute of Molecular and Translational Medicine and Department of Medicine I, Johannes Gutenberg University, Langenbeckstr.1, 55131 Mainz, Germany. Phone: 49.6131.177356; Fax: 49.6131.177357; E-mail: detlef.schuppan@unimedizin-mainz.de.
1. Friedman SL. Mechanisms of hepatic fibrogenesis. Gastroenterology. 2008;134(6):1655-1669.

2. Schuppan D, Afdhal NH. Liver cirrhosis. Lancet. 2008;371(9615):838-851.

3. Gao B, Bataller R. Alcoholic liver disease: pathogenesis and new therapeutic targets. Gastroenterology. 2011;141(5):1572-1585.

4. Tilg H, Moschen AR. Evolution of inflammation in nonalcoholic fatty liver disease: the multiple parallel hits hypothesis. Hepatology. 2010;52(5):1836-1846.

5. Bohinc BN, Diehl AM. Mechanisms of disease progression in NASH: new paradigms. Clin Liver Dis. 2012;16(3):549-565.

6. Popov Y, Schuppan D. Targeting liver fibrosis: strategies for development and validation of antifibrotic therapies. Hepatology. 2009;50(4):1294-1306.

7. Friedman SL. Evolving challenges in hepatic fibrosis. Nat Rev Gastroenterol Hepatol. 2010;7(8):425-436.

8. Schuppan D, Pinzani M. Anti-fibrotic therapy: lost in translation? J Hepatol. 2012;56(suppl 1):S66-S74.

9. Friedman SL, Sheppard D, Duffield JS, Violette S. Therapy for fibrotic diseases: nearing the starting line. Sci Transl Med. 2013;5(167):1-17.

10. Hinz B, et al. Recent developments in myofibroblast biology paradigms for connective tissue remodel- ing. Am J Pathol. 2012;180(4):1340-1355.

11. Dranoff JA, Wells RG. Portal fibroblasts: underappreciated mediators of biliary fibrosis. Hepatology. 2010;51(4):1438-1444.

12. Kisseleva T, Brenner DA. The phenotypic fate and functional role for bone marrow-derived stem cells in liver fibrosis. J Hepatol. 2012;56(4):965-972.

13. Gao B, et al. Innate immunity in alcoholic liver disease. Am J Physiol Gastrointest Liver Physiol. 2011; 300(4):G516-G525.

14. Chiang DAJ, Pritchard MT, Nagy LE. Obesity, diabetes mellitus, and liver fibrosis. Am J Physiol Gastrointest Liver Physiol. 2011;300(5):G697-G702.

15. Mehal W, Imaeda A. Cell death and fibrogenesis. Semin Liver Dis. 2010;30(3):226-231.

16. Wasmuth HE, Tacke F, Trautwein C. Chemokines in liver inflammation and fibrosis. Semin Liver Dis. 2010;30(3):215-225.

17. Forbes SJ, Newsome PN. New horizons for stem cell therapy in liver disease. J Hepatol. 2012;56(2):496-499.

18. Wynn TA, Ramalingam TR. Mechanisms of fibrosis: therapeutic translation for fibrotic disease. Nat Med. 2012;18(7):1028-1040.

19. Fallowfield JA. Therapeutic targets in liver fibrosis. Am J Physiol Gastrointest Liver Physiol. 2011;
300(5):G709-G715.

20. Schuppan D, Ruehl M, Somasundaram R, Hahn EG. Matrix as a modulator of hepatic fibrogenesis. Semin Liver Dis. 2001;21(3):351-372.

21. Fernandes DJ, Bonacci JV, Stewart AG. Extracellular matrix, integrins, and mesenchymal cell function in the airways. Curr Drug Targets. 2006;7(5):567-577.

22. Pozzi A, Voziyan PA, Hudson BG, Zent R. Regulation of matrix synthesis, remodeling and accumulation in glomerulosclerosis. Curr Pharm Des. 2009; 15(12):1318-1333.

23. Popov Y, et al. Macrophage-mediated phagocytosis of apoptotic cholangiocytes contributes to reversal of experimental biliary fibrosis. Am J Physiol Gastrointest Liver Physiol. 2010;298(3):G323-G334.

24. Friedman SL, Bansal MB. Reversal of hepatic fibrosis - fact or fantasy? Hepatology. 2006; 43(2 suppl 1):S82-S88.

25. Ellis EL, Mann DA. Clinical evidence for the regression of liver fibrosis. J Hepatol. 2012;56(5):1171-1180.

26. Issa $\mathrm{R}$, et al. Spontaneous recovery from micronodular cirrhosis: evidence for incomplete resolution associated with matrix cross-linking. Gastroenterology. 2004;126(7):1795-1808.

27. PopovY, et al. Tissue transglutaminase does not affect 
fibrotic matrix stability or regression of liver fibrosis in mice. Gastroenterology. 2011;140(5):1642-1652.

28. Popov Y, Schuppan D. Epithelial-to-mesenchymal transition in liver fibrosis: dead or alive? Gastroenterology. 2010;139(3):722-725

29. Huang CY, Ogawa R. Fibroproliferative disorders and their mechanobiology. Connect Tissue Res. 2012; 53(3):187-196

30. Puche JE, et al. A novel murine model to deplete hepatic stellate cells uncovers their role in amplifying liver damage in mice. Hepatology. 2013;57(1):340-350.

31. Wells RG. The role of matrix stiffness in hepatic stellate cell activation and liver fibrosis. J Clin Gastroenterol. 2005;39(4 suppl 2):S158-S161.

32. Eckes B, et al. Mechanical tension and integrin alpha 2 beta 1 regulate fibroblast functions. J Investig Dermatol Symp Proc. 2006;11(1):66-72.

33. Kisseleva T, et al. Myofibroblasts revert to an inactive phenotype during regression of liver fibrosis. Proc Natl Acad Sci U S A. 2012;109(24):9448-9453.

34. Troeger JS, et al. Deactivation of hepatic stellate cells during liver fibrosis resolution in mice. Gastroenterology. 2012;143(4):1073-1083.

35. Barczyk MM, Lu N, Popova SN, Bolstad AI, Gullberg D. Alpha11beta1 integrin-mediated MMP13-dependent collagen lattice contraction by fibroblasts: evidence for integrin-coordinated collagen proteolysis. J Cell Physiol. 2013;228(5):1108-1119.

36. Wang $\mathrm{P}$, et al. Mechanical stretch regulates the expression of matrix metalloproteinase in rheumatoid arthritis fibroblast-like synoviocytes. Connect Tissue Res. 2009;50(2):98-109.

37. Patsenker E, Stickel F. Role of integrins in fibrosing liver diseases. Am J Physiol Gastrointest Liver Physiol. 2011;301(3):G425-G434.

38. Goodman SL, Picard M. Integrins as therapeutic targets. Trends Pharmacol Sci. 2012;33(7):405-412.

39. Klein S, et al. HSC-specific inhibition of Rhokinase reduces portal pressure in cirrhotic rats without major systemic effects. J Hepatol. 2012; 57(6):1220-1227.

40. Neef $M$, et al. Oral imatinib treatment reduces early fibrogenesis but does not prevent progression in the long term. J Hepatol. 2006;44(1):167-175.

41. Ogawa S, et al. Anti-PDGF-B monoclonal antibody reduces liver fibrosis development. Hepatol Res. 2010;40(11):1128-1141.

42. Love KT, et al. Lipid-like materials for low-dose, in vivo gene silencing. Proc Natl Acad Sci U S A 2010;107(5):1864-1869.

43. Frank-Kamenetsky M, et al. Therapeutic RNAi targeting PCSK9 acutely lowers plasma cholesterol in rodents and LDL cholesterol in nonhuman primates. Proc Natl Acad Sci U S A. 2008;105(33):11915-11920.

44. Calvente CJ, Kim YO, Segal A, Kotelianski V, Novobrantseva T, Schuppan D. In vivo efficacy of C12200 lipid-like siRNA carriers to inhibit hepatic fibrosis [abstract]. Gastroenterology. 2012;142(5):S942.

45. Gonzalo T, et al. Local inhibition of liver fibrosis by specific delivery of a platelet-derived growth factor kinase inhibitor to hepatic stellate cells.J Pharmacol Exp Ther. 2007;321(3):856-865.

46. Poelstra K, Schuppan D. Targeted therapy of liver fibrosis/cirrhosis and its complications. J Hepatol. 2011;55(3):726-728.

47. Sato Y, et al. Resolution of liver cirrhosis using vitamin A-coupled liposomes to deliver siRNA against a collagen-specific chaperone. Nat Biotechnol. 2008;26(4):431-442.

48. Bansal R, Prakash J, Post E, Beljaars L, Schuppan D, Poelstra K. Novel engineered targeted interferongamma blocks hepatic fibrogenesis in mice. Hepatology. 2011;54(2):586-596.

49. Pasquetto MV, Vecchia L, Covini D, Digilio R, Scotti C. Targeted drug delivery using immunoconjugates: principles and applications. J Immunother. 2011;34(9):611-628.

50. Malhi H, Gores GJ. Molecular mechanisms of lipo- toxicity in nonalcoholic fatty liver disease. Semin Liver Dis. 2008;28(4):360-369.

51. Jiang JX, et al. Reduced nicotinamide adenine dinucleotide phosphate oxidase 2 plays a key role in stellate cell activation and liver fibrogenesis in vivo. Gastroenterology. 2010;139(4):1375-1384.

52. Jiang JX, Mikami K, Venugopal S, Li Y, Torok NJ. Apoptotic body engulfment by hepatic stellate cells promotes their survival by the JAK/STAT and Akt/NF-kappaB-dependent pathways. J Hepatol. 2009;51(1):139-148.

53. Canbay A, et al. Cathepsin B inactivation attenuates hepatic injury and fibrosis during cholestasis. J Clin Invest. 2003;112(2):152-159.

54. Canbay A, Feldstein A, Baskin-Bey E, Bronk SF, Gores GJ. The caspase inhibitor IDN-6556 attenuates hepatic injury and fibrosis in the bile duct ligated mouse. J Pharmacol Exp Ther. 2004;308(3):1191-1196.

55. Richardson MM, et al. Progressive fibrosis in nonalcoholic steatohepatitis: association with altered regeneration and a ductular reaction. Gastroenterology. 2007;133(1):80-90.

56. Jung Y, et al. Accumulation of hedgehog-responsive progenitors parallels alcoholic liver disease severity in mice and humans. Gastroenterology. 2008; 134(5):1532-1543

57. Syn WK, et al. Hedgehog-mediated epithelial-tomesenchymal transition and fibrogenic repair in nonalcoholic fatty liver disease. Gastroenterology. 2009;137(4):1478-1488

58. Jung YM, et al. Signals from dying hepatocytes trigger growth of liver progenitors. Gut. 2010; 59(5):655-665.

59. Syn WK, et al. NKT-associated hedgehog and osteopontin drive fibrogenesis in non-alcoholic fatty liver disease. Gut. 2012;61(9):1323-1329.

60. Berenguer M, Schuppan D. Progression of liver fibrosis in post-transplant hepatitis C: mechanisms, assessment and treatment. J Hepatol. 2012; pii:S0168-8278(12)00962-2.

61. Wang B, et al. Role of alphavbeta6 integrin in acute biliary fibrosis. Hepatology. 2007;46(5):1404-1412.

62. Patsenker E, Popov Y, Stickel F, Jonczyk A, Goodman SL, Schuppan D. Inhibition of integrin alphavbeta6 on cholangiocytes blocks transforming growth factor-beta activation and retards biliary fibrosis progression. Gastroenterology. 2008;135(2):660-670.

63. Popov Y, et al. Integrin alphavbeta6 is a marker of the progression of biliary and portal liver fibrosis and a novel target for antifibrotic therapies. J Hepatol. 2008;48(3):453-464.

64. Omenetti A, et al. Hedgehog signaling regulates epithelial-mesenchymal transition during biliary fibrosis in rodents and humans. J Clin Invest. 2008; 118(10):3331-3342

65. Philips GM, et al. Hedgehog signaling antagonist promotes regression of both liver fibrosis and hepatocellular carcinoma in a murine model of primary liver cancer. PLoS One. 2011;6(9):E23943.

66. Thabut D, Shah V. Intrahepatic angiogenesis and sinusoidal remodeling in chronic liver disease: new targets for the treatment of portal hypertension? J Hepatol. 2010;53(5):976-980.

67. Tugues S, et al. Antiangiogenic treatment with sunitinib ameliorates inflammatory infiltrate, fibrosis, and portal pressure in cirrhotic rats. Hepatology. 2007;46(6):1919-1926.

68. Thabut D, et al. Complementary vascular and matrix regulatory pathways underlie the beneficial mechanism of action of sorafenib in liver fibrosis. Hepatology. 2011;54(2):573-585.

69. Kang DH, Hughes J, Mazzali M, Schreiner GF, Johnson RJ. Impaired angiogenesis in the remnant kidney model: II. Vascular endothelial growth factor administration reduces renal fibrosis and stabilizes renal function. J Am Soc Nephrol. 2001;12(7):1448-1457.

70. Stockmann C, et al. Loss of myeloid cell-derived vascular endothelial growth factor accelerates fibrosis.
Proc Natl Acad Sci U S A. 2010;107(9):4329-4334.

71. Patsenker E, et al. Pharmacological inhibition of integrin alphavbeta3 aggravates experimental liver fibrosis and suppresses hepatic angiogenesis. Hepatology. 2009;50(5):1501-1511.

72. Yang L, Popov Y, Schuppan D, Ordog T, Mukhopadhyay D, Shah V. Opposing roles of VEGF during liver fibrogenesis and fibrosis resolution [abstract]. Hepatology. 2012;56(suppl 4):768A. doi:10.1002/hep.26040.

73. Wilson MS, Wynn TA. Pulmonary fibrosis: pathogenesis, etiology and regulation. Mucosal Immunol. 2009;2(2):103-121.

74. Wynn TA, Barron L. Macrophages: master regulators of inflammation and fibrosis. Semin Liver Dis. 2010;30(3):245-257

75. Barron L, Wynn TA. Fibrosis is regulated by Th2 and Th17 responses and by dynamic interactions between fibroblasts and macrophages. Am J Physiol Gastrointest Liver Physiol. 2011;300(5):G723-G728.

76. Lee CG, et al. Interleukin-13 induces tissue fibrosis by selectively stimulating and activating transforming growth factor beta(1). J Exp Med. 2001; 194(6):809-821.

77. Mentink-Kane MM, et al. Accelerated and progressive and lethal liver fibrosis in mice that lack interleukin (IL)-10, IL-12p40, and IL-13Ralpha2. Gastroenterology. 2011;141(6):2200-2209.

78. Wynn TA, et al. An IL-12-based vaccination method for preventing fibrosis induced by schistosome infection. Nature. 1995;376(6541):594-596.

79. Kamal SM, et al. Progression of fibrosis in hepatitis $\mathrm{C}$ with and without schistosomiasis: Correlation with serum markers of fibrosis. Hepatology. 2006;43(4):771-779.

80. Meng F, et al. Interleukin-17 signaling in inflammatory, kupffer cells, and hepatic stellate cells exacerbates liver fibrosis in mice. Gastroenterology. 2012;143(3):765-776

81. Wilson MS, et al. Bleomycin and IL-1beta-mediated pulmonary fibrosis is IL-17A dependent. J Exp Med. 2010;207(3):535-552.

82. Gao B, Waisman A. Th17 cells regulate liver fibrosis by targeting multiple cell types: many birds with one stone. Gastroenterology. 2012;143(3):536-539.

83. Gao B, Radaeva S. Natural killer and natural killer T cells in liver fibrosis. Biochim Biophys Acta. 2012;pii:S0925-4439(12)00217-7.

84. Park O, et al. Diverse roles of invariant natural killer $T$ cells in liver injury and fibrosis induced by carbon tetrachloride. Hepatology. 2009;49(5):1683-1694.

85. Lynch L, et al. Adipose tissue invariant NKT cells protect against diet-induced obesity and metabolic disorder through regulatory cytokine production. Immunity. 2012;37(3):574-587.

86. Marra F, Aleffi S, Galastri S, Provenzano A. Mononuclear cells in liver fibrosis. Semin Immunopathol. 2009;31(3):345-358

87. Gabrilovich DI, Nagaraj S. Myeloid-derived suppressor cells as regulators of the immune system. Nat Rev Immunol. 2009;9(3):162-174.

88. Karlmark KR, et al. Hepatic recruitment of the inflammatory Gr1(+) monocyte subset upon liver injury promotes hepatic fibrosis. Hepatology. 2009; 50(1):261-274.

89. Zimmermann HW, et al. Functional contribution of elevated circulating and hepatic non-classical CD14CD16 monocytes to inflammation and human liver fibrosis. PLoS One. 2010;5(6):E11049.

90. Saiman Y, Friedman SL. The role of chemokines in acute liver injury. Front Physiol. 2012;3:213.

91. Wasmuth HE, et al. Antifibrotic effects of CXCL9 and its receptor CXCR3 in livers of mice and humans. Gastroenterology. 2009;137(1):309-319.

92. Sahin $\mathrm{H}$, et al. Chemokine $\mathrm{Cxcl} 9$ attenuates liver fibrosis-associated angiogenesis in mice. Hepatology. 2012;55(5):1610-1619.

93. Karlmark KR, et al. The fractalkine receptor CX(3) 
CR1 protects against liver fibrosis by controlling differentiation and survival of infiltrating hepatic monocytes. Hepatology. 2010;52(5):1769-1782.

94. Gordon S, Martinez FO. Alternative activation of macrophages: mechanism and functions. Immunity. 2010;32(5):593-604.

95. Murray PJ, Wynn TA. Protective and pathogenic functions of macrophage subsets. Nat Rev Immunol. 2011;11(11):723-737.

96. Ramachandran P, et al. Differential Ly-6C expression identifies the recruited macrophage phenotype, which orchestrates the regression of murine liver fibrosis. Proc Natl Acad Sci U S A. 2012; 109(46):E3186-E3195

97. Olefsky JM, Glass CK. Macrophages,inflammation, and insulin resistance. Annu Rev Physiol. 2010; 72:219-246.

98. Barry-Hamilton $\mathrm{V}$, et al. Allosteric inhibition of lysyl oxidase-like- 2 impedes the development of a pathologic microenvironment. Nat Med. 2010; 16(9):1009-1017.

99. Yang L, Seki E. Toll-like receptors in liver fibrosis: cellular crosstalk and mechanisms. Front Physiol. 2012;3:138.

100. Seki E, et al. TLR4 enhances TGF-beta signaling and hepatic fibrosis. Nat Med. 2007;13(11):1324-1332.

101. Tidswell M, LaRosa SP. Toll-like receptor-4 antagonist eritoran tetrasodium for severe sepsis. Expert Rev Anti Infect Ther. 2011;9(5):507-520.

102.Jeong WI, et al. Suppression of innate immunity (natural killer cell/interferon-gamma) in the advanced stages of liver fibrosis in mice. Hepatology. 2011 53(4):1342-1351.

103. Gabele E, et al. Role of TLR9 in hepatic stellate cells and experimental liver fibrosis. Biochem Biophys Res Commun. 2008;376(2):271-276.

104.Zaldivar MM, et al. CXC chemokine ligand 4 (CXCL4) is a platelet-derived mediator of experimental liver fibrosis. Hepatology. 2010;51(4):1345-1353.

105. Berres ML, et al. Antagonism of the chemokine $\mathrm{Ccl} 5$ ameliorates experimental liver fibrosis in mice. J Clin Invest. 2010;120(11):4129-4140.

106. Seki E, et al. CCR1 and CCR5 promote hepatic fibrosis in mice. J Clin Invest. 2009;119(7):1858-1870.

107. Sekiya Y, Ogawa T, Yoshizato K, Ikeda K, Kawada N. Suppression of hepatic stellate cell activation by microRNA-29b. Biochem Biophys Res Commun. 2011;412(1):74-79.

108. Murakami Y, et al. The progression of liver fibrosis is related with overexpression of the miR-199 and 200 families. PLoS One. 2011;6(1):e16081.

109. Wang XW, Heegaard NH, Orum H. MicroRNAs in liver disease. Gastroenterology. 2012;142(7):1431-1443.

110.Tedgui A, Mallat Z. Cytokines in atherosclerosis Pathogenic and regulatory pathways. Physiol Rev. 2006;86(2):515-581.

111. Olinga P, Schuppan D. Precision-cut liver slices: a tool to model the liver ex vivo. J Hepatol. 2013 pii:S0168-8278(13)00052-4

112.Marcellin P, et al. Regression of cirrhosis during treatment with tenofovir disoproxil fumarate for chronic hepatitis B: a 5-year open-label follow-up study. Lancet. 2013;381(9865):468-475.

113. Huang HJ, et al. A 7 gene signature identifies the risk of developing cirrhosis in patients with chronic hepatitis C. Hepatology. 2007;46(2):297-306.

114. Castera L. Noninvasive methods to assess liver disease in patients with hepatitis B or C. Gastroenterol ogy. 2012;142(6): 1293-1302.

115. Gabrielli GB, Casaril M, Stanzial AM, Colombari R, Pasino M, Corrocher R. Liver stellate cells and aminoterminal peptide of type III procollagen in chronic hepatitis $\mathrm{C}$ treated with interferon. Hepato Gastroenterol. 2003;50(54):2017-2021.

116. Ferraioli G, et al. Accuracy of real-time shear wave elastography for assessing liver fibrosis in chronic hepatitis C: A pilot study. Hepatology. 2012;56(6):2125-2133.

117. Huwart L, et al. Magnetic resonance elastography for the noninvasive staging of liver fibrosis. Gastroenterology. 2008;135(1):32-40.

118. Parkes J, Guha IN, Roderick P, Rosenberg W. Performance of serum marker panels for liver fibrosis in chronic hepatitis C. J Hepatol. 2006;44(3):462-474.

119. Manning DS, Afdhal NH. Diagnosis and quantitation of fibrosis. Gastroenterology. 2008;134(6):1670-1681.

120.Guha IN, Myers RP, Patel K, Talwalkar JA. Biomarkers of liver fibrosis: what lies beneath the receiver operating characteristic curve? Hepatology. 2011;54(4):1454-1462.

121. Martinez SM, Crespo G, Navasa M, Forns X. Noninvasive assessment of liver fibrosis. Hepatology. 2011;53(1):325-335.

122. Poynard T, et al. Longitudinal assessment of histology surrogate markers (FibroTest-ActiTest) during lamivudine therapy in patients with chronic hepatitis B infection. Am J Gastroenterol. 2005; 100(9):1970-1980.

123. Mayo MJ, et al. Prediction of clinical outcomes in primary biliary cirrhosis by serum enhanced liver fibrosis assay. Hepatology. 2008;48(5):1549-1557.

124. Parkes J, et al. Enhanced liver fibrosis test can predict clinical outcomes in patients with chronic liver disease. Gut. 2010;59(9):1245-1251.

125. Fontana RJ, et al. Serum fibrosis markers are associated with liver disease progression in non-responder patients with chronic hepatitis C. Gut. 2010; 59(10):1401-1409.

126. Ripoll C, et al. Hepatic venous pressure gradient predicts clinical decompensation in patients with compensated cirrhosis. Gastroenterology. 2007; 133(2):481-488.

127. Kim MY, et al. Hepatic vein arrival time as assessed by contrast-enhanced ultrasonography is useful for the assessment of portal hypertension in compensated cirrhosis. Hepatology. 2012;56(3):1053-1062.

128.Nista EC, et al. 13C-breath tests in the study of microsomal liver function. Eur Rev Med Pharmacol Sci. 2004;8(1):33-46.

129. Makowski MR, et al. Assessment of atherosclerotic plaque burden with an elastin-specific magnetic resonance contrast agent. Nat Med. 2011;17(3):383-388.

130. Kwong GA, et al. Mass-encoded synthetic biomarkers for multiplexed urinary monitoring of disease. Nat Biotechnol. 2012;31(1):63-70.

131. Kornek M, Schuppan D. Microparticles: Modulators and biomarkers of liver disease. J Hepatol. 2012; 57(5):1144-1146.

132. Nelson DR, et al. Long-term interleukin 10 therapy in chronic hepatitis $C$ patients has a proviral and anti-inflammatory effect. Hepatology. 2003; 38(4):859-868.

133.Vilar Gomez E, Gra Oramas B, Soler E, Llanio Navarro R, Ruenes Domech C. Viusid, a nutritional supplement, in combination with interferon alpha$2 \mathrm{~b}$ and ribavirin in patients with chronic hepatitis C. Liver Int. 2007;27(2):247-259.

134.Colmenero J, et al. Effects of losartan on hepatic expression of nonphagocytic NADPH oxidase and fibrogenic genes in patients with chronic hepatitis C. Am J Physiol Gastrointest Liver Physiol. 2009; 297(4):G726-G734.

135. McHutchison J, et al. Farglitazar lacks antifibrotic activity in patients with chronic hepatitis $\mathrm{C}$ infection. Gastroenterology. 2010;138(4):1365-1373.

136. Poynard T, et al. Impact of pegylated interferon alfa- $2 b$ and ribavirin on liver fibrosis in patients with chronic hepatitis C. Gastroenterology. 2002; 122(5):1303-1313.

137. Pockros PJ, et al. Final results of a double-blind, placebo-controlled trial of the antifibrotic efficacy of interferon-gamma1b in chronic hepatitis $\mathrm{C}$ patients with advanced fibrosis or cirrhosis. Hepatology. 2007;45(3):569-578.

138.Di Bisceglie AM, et al. Prolonged therapy of advanced chronic hepatitis $C$ with low-dose peginterferon. NEngl J Med. 2008;359(23):2429-2441.
139. Liu P, et al. Clinical observation of salvianolic acid B in treatment of liver fibrosis in chronic hepatitis B. World J Gastroenterol. 2002;8(4):679-685.

140.Kweon YO, et al. Decreasing fibrogenesis: an immunohistochemical study of paired liver biopsies following lamivudine therapy for chronic hepatitis B. J Hepatol. 2001;35(6):749-755.

141. Poupon RE, Balkau B, Eschwege E, Poupon R. A multicenter, controlled trial of ursodiol for the treatment of primary biliary cirrhosis. UDCA-PBC Study Group. NEngl J Med. 1991;324(22):1548-1554.

142. Corpechot C, Carrat F, Bonnand AM, Poupon RE, Poupon R. The effect of ursodeoxycholic acid therapy on liver fibrosis progression in primary biliary cirrhosis. Hepatology. 2000;32(6):1196-1199.

143. Kim MY, et al. Beneficial effects of candesartan, an angiotensin-blocking agent, on compensated alcoholic liver fibrosis - A randomized open-label controlled study. Liver Int. 2012;32(6):977-987.

144. Belfort R, et al. A placebo-controlled trial of pioglitazone in subjects with nonalcoholic steatohepatitis. NEngl J Med. 2006;355(22):2297-2307.

145.Aithal GP, et al. Randomized, placebo-controlled trial of pioglitazone in nondiabetic subjects with nonalcoholic steatohepatitis. Gastroenterology. 2008; 135(4):1176-1184.

146. Sanyal AJ, et al. Pioglitazone, vitamin E, or placebo for nonalcoholic steatohepatitis. $N$ Engl J Med. 2010;362(18):1675-1685.

147. Ratziu $\mathrm{V}$, et al. Long-term efficacy of rosiglitazone in nonalcoholic steatohepatitis: results of the fatty liver improvement by rosiglitazone therapy (FLIRT 2) extension trial. Hepatology. 2010;51(2):445-453.

148.Zein CO, et al. Pentoxifylline improves nonalcoholic steatohepatitis: a randomized placebo-controlled trial. Hepatology. 2011;54(5):1610-1619.

149. Torres DM, Jones FJ, Shaw JC, Williams CD, Ward JA, Harrison SA. Rosiglitazone versus rosiglitazone and metformin versus rosiglitazone and losartan in the treatment of nonalcoholic steatohepatitis in humans: a 12-month randomized, prospective, open- label trial. Hepatology. 2011;54(5):1631-1639.

150. Ratziu V, et al. A randomized controlled trial of high-dose ursodesoxycholic acid for nonalcoholic steatohepatitis. J Hepatol. 2011;54(5):1011-1019.

151. Harrison SA, Torgerson S, Hayashi P, Ward J, Schenker S. Vitamin E and vitamin C treatment improves fibrosis in patients with nonalcoholic steatohepatitis. Am J Gastroenterol. 2003;98(11):2485-2490.

152. Chavez-Tapia NC, Tellez-Avila FI, BarrientosGutierrez T, Mendez-Sanchez N, Lizardi-Cervera J, Uribe M. Bariatric surgery for non-alcoholic steatohepatitis in obese patients. Cochrane Database Syst Rev. 2010;(1):CD007340

153. Dufour JF, DeLellis R, Kaplan MM. Reversibility of hepatic fibrosis in autoimmune hepatitis. Ann Intern Med. 1997;127(11):981-985.

154.Ferreira AR, Roquete ML, Toppa NH, de Castro LP, Fagundes ED, Penna FJ. Effect of treatment of hepatic histopathology in children and adolescents with autoimmune hepatitis. J Pediatr Gastr Nutr. 2008;46(1):65-70

155. Raghu G, et al. Treatment of idiopathic pulmonary fibrosis with etanercept: an exploratory, placebocontrolled trial. Am J Respir Crit Care Med. 2008; 178(9):948-955.

156.Demedts M, et al. High-dose acetylcysteine in idiopathic pulmonary fibrosis. $N$ Engl J Med. 2005; 353(21):2229-2242.

157. Couluris M, Kinder BW, Xu P, Gross-King M, Krischer J, Panos RJ. Treatment of idiopathic pulmonary fibrosis with losartan: a pilot project. Lung. 2012;190(5):523-527.

158. Daniels CE, et al. Imatinib treatment for idiopathic pulmonary fibrosis: Randomized placebo-controlled trial results. Am J Respir Crit Care Med. 2010; 181(6):604-610

159. Raghu G, et al. Artemis-Ipf: a placebo-controlled 
trial of ambrisentan in idiopathic pulmonary fibrosis. Am J Respir Crit Care Med. 2012;186(1):88-95.

160. Noble PW, et al. Pirfenidone in patients with idiopathic pulmonary fibrosis (CAPACITY): two randomised trials. Lancet. 2011;377(9779):1760-1769.

161. Taniguchi $\mathrm{H}$, et al. Pirfenidone in idiopathic pulmonary fibrosis. Eur Respir J. 2010;35(4):821-829.

162. Richeldi L, et al. Efficacy of a tyrosine kinase inhibitor in idiopathic pulmonary fibrosis. $N$ Engl J Med. 2011;365(12):1079-1087.

163. Duffield JS, Lupher ML Jr. PRM-151 (recombinant human serum amyloid $\mathrm{P} /$ pentraxin 2 ) for the treatment of fibrosis. Drug News Perspect. 2010;
23(5):305-315.

164. Miossec P, Kolls JK. Targeting IL-17 and TH17 cells in chronic inflammation. Nat Rev Drug Discov. 2012;11(10):763-776

165. Gilliam BL, Riedel DJ, Redfield RR. Clinical use of CCR5 inhibitors in HIV and beyond. J Transl Med. 2011;9(suppl 1):S9.

166.de Nigris F, Schiano C, Infante T, Napoli C. CXCR4 inhibitors: tumor vasculature and therapeutic challenges. Recent Pat Anti-Canc. 2012;7(3):251-264.

167. Fang F, et al. The adipokine adiponectin has potent anti-fibrotic effects mediated via adenosine monophosphate-activated protein kinase: novel target for fibrosis therapy. Arthritis Res Ther. 2012;14(5):R229.

168.Popov Y, Patsenker E, Bauer M, Niedobitek E, Schulze-Krebs A, Schuppan D. Halofuginone induces matrix metalloproteinases in rat hepatic stellate cells via activation of p38 and NFkappaB. J Biol Chem. 2006;281(22):15090-15098.

169.Peng $Z$, et al. Adenosine signaling contributes to ethanol-induced fatty liver in mice. J Clin Invest. 2009;119(3):582-594.

170.Zhang Z, Wang F. Stem cell therapies for liver failure and cirrhosis [published online ahead of print January 23, 2013]. J Hepatol. doi:10.1016/j. jhep.2013.01.018. 